\title{
SUR L'HOLONOMIE DES VARIÉTÉS PSEUDO-RIEMANNIENNES DE SIGNATURE $(2,2+n)$
}

\author{
A. IKEMAKHen
}

\begin{abstract}
In this paper, we determinate a class of possible restricted holonomy groups for a non-irreducible indecomposable pseudoriemannian manifold with signature $(2,2+n)$. In particular, we deduce that which associated to symmetric spaces; and give some examples of such spaces. Finally, we construct some examples of metrics whose restricted holonomy groups are not closed.
\end{abstract}

\section{Introduction}

Soit $M$ une variété connexe de dimension $m$. Soit $D$ une connection affine sans torsion sur le fibré tangent de $M$ et soit $\theta$ un point de base pour $M$. Le groupe d'holonomie en $\theta$, noté $H_{\theta}$, est le sous-groupe de $G L\left(T_{\theta} M\right)$ engendré par le transport parallèle le long des lacets basés en $\theta$. Si on change le point $\theta$ en un autre point $\delta$, on montre facilement que $H_{\theta}$ et $H_{\delta}$ sont conjugués. Donc, par abus de language, on parle sur $H$ comme groupe d'holonomie et l'algèbre de Lie $\mathcal{H}$ de $H$ comme algèbre d'holonomie de $(M, D)$.

Pour simplifier, nous nous restreindrons ici au problème "local", en ne considérant que le groupe d'holonomie "restreint", qui est engendré par le transport parallèle le long des lacets homotopes au lacet constant. (Pour alléger les notations, dans le cours du texte nous omettrons parfois le mot restreint, mais nous n'étudions en fait ici que ce cas.) Les invariants de ce groupe correspondent aux tenseurs parallèles sur $M$ et la classification des groupes linéaires qui peuvent apparaître ainsi est un problème important de géométrie différentielle. Ainsi la question de base de la théorie est la suivante:

Keywords. Restricted holonomy group, indecomposable pseudo-Riemannian manifold, cohomologie of Lie algebra.

1991 Mathematics subject classifications: Primary: 53B05, 53C10. 
A une conjuguaison près, quels sont tous les sous-groupes connexes de $H$ de $G L(m, \mathbb{R})$ qui peuvent être réalisés comme groupe d'holonomie d'une connection affine sans torsion sur une variété de dimension $m$ ?

L'outil clé de la réponse à cette question, dans le cas d'une variété pseudo-riemannienne, est le classique Théoréme de G. De Rham (pour le cas riemannien, voir le chapitre 10 dans [7] par exemple), généralisé par $\mathrm{H}$. Wu (dans le cas pseudo-riemannien, voir [25], [26]). Si la représentation du groupe d'holonomie $H$ dans $T_{\theta} M$ est décomposable en une somme directe orthogonale de représentations dans des sous-espaces $E_{i}$ $(i=1, \ldots, r)$ de $T_{\theta} M$, non dégénérés pour $g_{\theta}$, alors, au moins localement au voisinage de $\theta$, il existe une décomposition de $M$ en produit riemannien de sous-variétés pseudo-riemanniennes $M_{i}$ telles que $E_{i}$ soit l'espace tangent à $M_{i}$, et si $H_{i}$ est le groupe d'holonomie de $M_{i}$, alors $H$ est en fait le produit des $H_{i}$, et $H_{i}$ est l'image de $H$ dans $G L\left(E_{i}\right)$.

On est donc ainsi ramené, dans ce cas, à étudier les groupes d'holonomie "indécomposables", c'est à dire tels qu'il n'existe pas de sousespace non-dégénéré propre dans $T_{\theta} M$, invariant par $H$. Dans le cas riemannien, on est donc ramené au cas où la représentation de $H$ dans $T_{\theta} M$ est irréductible. Par contre, dans le cas pseudo-riemannien, il peut y avoir encore des sous-espaces invariants pour la représentation de $H$, à condition que ces sous-espaces soient dégénérés pour $g_{\theta}$.

En utilisant deux critères qui dérivent du Théorème d'AmbroseSinger [2], M. Berger a donné dans [5] une liste de groupes d'holonomie possibles dont la représentation est irréductible, pour une connection affine non localement symétrique. Sa classification est divisée en trois parties:

(1) La première liste est constituée de tous les sous-groupes irréductibles $H \subseteq S O_{0}(m)$, c'est à dire ceux qui peuvent être associés à une métrique définie positive. Après sept ans, J. Simons dans [24] a démontré que cette liste est celle des sous-groupes de $S O_{0}(m)$ qui agit transitivement sur la sphère unité de $\mathbb{R}^{m}$, en se basant sur les travaux de D. Montgomery et H. Samelson [19]. D'autre part, il a fallu beaucoup de progès pour réaliser ces groupes comme holonomies de variétés riemanniennes. Nous mentionnons dans ce contexte les travaux de Calabi, Alekseevskii, Brow-Gray, Salamon et Bryant [7], [15], [1], [8], [9], [21] et [11].

(2) La deuxième liste est constituée de tous les sous-groupes irréductibles $H \subseteq S O_{0}(p, m-p)$, pour $p \neq 0$. C'est à dire ceux qui peuvent être associés à une métrique de signature quelconque $(p, m-p)$ non définie positive. Dans ce cas la question est ce que ces condidats sont réalisés ou non comme holonomies de métriques pseudo-riemanniennes est résolue (voir $[\mathbf{9}]$ et $[\mathbf{1 0}]$ ). 
(3) La troixième liste est constituée de tous les sous-groupes irréductibles $H \subseteq G L(m, \mathbb{R})$ mais $H$ est non inclu dans $S O_{0}(p, m-p)$, c'est à dire ceux qui peuvent être associés à une connection affine sans torsion et ne laissent invariante aucune 2-forme bilinéaire symétrique non dégénérée. La liste de Berger dans ce cas est incomplète. Bryant l'a complété par d'autres sous-groupes et il les a appelé les groupes d'holonomie "exotiques" (voir la revue de synthèse [10]). La réalisation de ses groupes comme groupes d'holonomie a été faite ces dernières années. Nous mentionnons dans ce contexte les travaux de R. L. Bryant, S. Merkulov, L. Schwachhöfer [10], [16], [18], [22] et [23].

Malgré tous ces travaux, le cas des groupes d'holonomie indécomposables et non-irréductibles n'a été que peu abordé jusqu'à présent, et même la classification des espaces symétriques pseudo-riemanniens n'est pas terminée. Cette classification n'est faite que dans le cas lorentzien (signature $(1, m-1)$, voir M. Cahen et N. Wallach [14]) et dans le cas de la signature $(2, m-2)$, voir M. Cahen et M. Parker [12], [13] et [20].

L'auteur et L. Berard Bergery ont commencé l'étude des groupes d'holonomie indécomposables et non-irréductibles par le cas lorentzien, dans les articles $[\mathbf{3}]$ et $[\mathbf{1 7}]$. Récemment, ils ont étudié le cas "nentre" de la signature $(n, n)$ dans l'article [4]. Le but de ce papier est d'étendre les résultats de $[\mathbf{3}],[\mathbf{1 7}]$ et [4] pour les variétés pseudo-riemanniennes de signature $(2,2+n)$. Le résultat principal est la détérmination d'une classe de sous-groupes connexes de $S O_{0}(2,2+n)$ qui peuvent être holonomie d'une variété pseudo-riemannienne de signature $(2,2+n)$ indécomposable non irréductible. Plus précisement, nous déterminons une classe de sous-groupes connexes de $S O_{0}(2,2+n)$ qui ne laissent invariant aucun sous-espace non trivial non dégénéré, mais qui laissent invariant au moins un plan totalement isotrope (Théorème 3.19). La démonstration de ce théorème repose essentiellement sur le premier Lemme de Whitehead. Au paragraphe 4, on rappelle les résultats généraux de Cahen et Parker pour les espaces pseudo-riemanniens symétriques et on précise les groupes d'holonomie restreints possibles de ces espaces, en comparant aux résultats du Théorème 3.19 (voir les Propositions 4.21 et 4.24). Dans le paragraphe 5, on montre que si l'holonomie d'une métrique pseudoriemannienne laisse invariant un sous-espace isotrope maximal, alors il existe un système de coordonnées dans lequel la métrique a une forme réduite (Théorème 5.27). Enfin, dans le paragraphe 6, en se basant sur les résultats du paragraphe 5 , nous construisons d'autres exemples de métriques dont les groupes d'holonomie restreints sont non fermés (Théorème 6.28).

Je tiens à remercier les professeurs L. Berard Bergery et M. Sarih pour les nombreuses suggestions qu'ils m'ont faites au cours de ce travail. 


\section{Une classification algèbrique}

Nous rappelons d'abord les définitions principales.

Définition 1. Soit $V$ un espace vectoriel reél, muni d'une forme bilinère symétrique non dégénéré. Un sous-groupe connexe $H$ de $G L(V)$ est dit indécomposable s'il ne laisse invariant aucun sous-espace propre non dégénéré de $V$. Une sous-algèbre $\mathcal{H}$ de $g l(V)$ est dite indécomposable si son sous-groupe connexe associé l'est.

Définition 2. Une variété pseudo-riemannienne $(M, g)$ connexe est dite irréductible si sa représentation d'holonomie restreint $H_{\theta}^{\circ}$ en un point $\theta \in M$ est irréductible.

$(M, g)$ est dite indécomposable si $H_{\theta}^{\circ}$ ne laisse invariant aucun sousespace propre non dégénéré de $T_{\theta} M$.

Maintenant, soit $(M, g)$ une variété pseudo-riemannienne connexe de dimension $m=n+2 r,(r \geq 2)$ avec $g$ de signature $(r, n+r)$. On suppose que $(M, g)$ est indécomposable et non irréductible donc la représentation d'holonomie restreint $H_{\theta}^{\circ}$ en un point $\theta \in M$ ne laisse invariant aucun sous-espace propre non dégénéré de $T_{\theta} M$ et laisse invariant au moins un sous-espace propre $E$ dégénéré de $T_{\theta} M$, donc aussi son orthogonal $E^{\perp}$ et l'intersection $E \cap E^{\perp}$ qui est un sous-espace totalement isotrope. Par conséquent $H=H_{\theta}^{\circ}$ est un sous-groupe connexe de $S O\left(T_{\theta} M, g_{\theta}\right)$ qui laisse invariant un sous-espace $\Re$ totalement isotrope de dimension $\leq r$, mais ne laisse invariant aucun sous-espace propre non dégénéré de $T_{\theta} M$.

Nous classifierons certaines possibilités de tels sous-groupes $H$ qui laissent invariant un sous-espace totalement isotrope $\Re$ maximal. On notera $\mathcal{H}$ l'algèbre de Lie de $H$.

Soit $\left(e_{1}, \ldots, e_{m}\right)$ la base canonique de $\mathbb{R}^{m}$ et considérons sur $\mathbb{R}^{m}$ la 2-forme symétrique \langle\rangle de signature $(r, n+r)$ définit par:

$$
\left\langle\left(x_{1}, \ldots, x_{m}\right),\left(y_{1}, \ldots, y_{m}\right)\right\rangle=\sum_{i=1}^{r}\left(x_{i} y_{n+r+i}+y_{i} x_{n+r+i}\right)-\sum_{j=r+1}^{r+n} x_{j} y_{j}
$$

Si on identifie $\left(T_{\theta} M, g_{\theta}\right)$ à $\left(\mathbb{R}^{m},\langle\rangle\right)$, alors $\Re \simeq \mathbb{R} e_{1}+\cdots+\mathbb{R} e_{r}$. Et si on note $\mathbf{G}$ le sous-groupe connexe de $S O(\langle\rangle)$ engendré par les matrices qui laissent invariant $\Re$, alors l'algébre de Lie $\mathcal{G}$ de $\mathbf{G}$ est formée par les matrices "bloc":

$$
\left(\begin{array}{ccc}
U & { }^{t} X & B \\
0 & A & X \\
0 & 0 & -{ }^{t} U
\end{array}\right)
$$


où $U \in g l(r, \mathbb{R}), X \in \mathbb{R}^{r n}$ vu comme une matrice $(r, n),{ }^{t} X$ sa matrice transposée, $A \in \operatorname{so}(n, \mathbb{R}), B \in \operatorname{so}(r, \mathbb{R})$. Pour simplifier les notations, on identifie l'élément ci-dessus à $(U, A, X, B)$ et $\mathcal{G}$ à $\mathcal{U} \oplus \mathcal{K} \oplus \mathcal{N} \oplus \mathcal{B}$, avec

$\mathcal{B}=\{(0,0,0, B) / B \in \operatorname{so}(r, \mathbb{R})\}$ un idéal abélien de $\mathcal{G}$,

$\mathcal{N} \oplus \mathcal{B}=\left\{(0,0, X, B) / X \in \mathbb{R}^{r n}, B \in \operatorname{so}(r, \mathbb{R})\right\}$ un idéal de $\mathcal{G}$,

$\mathcal{K}=\{(0, A, 0,0) / A \in \operatorname{so}(n, \mathbb{R})\}$ une sous-algèbre isomorphe à $s o(n, \mathbb{R})$ et $\mathcal{U}=\{(U, 0,0,0) / U \in g l(r, \mathbb{R})\}$ une sous-algèbre isomorphe à $g l(r, \mathbb{R})$. Si on identifie $X=\left(X_{1}, \ldots, X_{r}\right)$ à $\left(\begin{array}{c}X_{1} \\ \vdots \\ X_{r}\end{array}\right)$, le crochet dans $\mathcal{G}$ est défini par:

(1) $\left[(U, A, X, B),\left(U^{\prime}, A^{\prime}, X^{\prime}, B^{\prime}\right)\right]=\left(\left[U, U^{\prime}\right],\left[A, A^{\prime}\right],(\widetilde{U}+\tilde{A}) X^{\prime}\right.$

$$
\left.-\left(\widetilde{U}^{\prime}+\tilde{A}^{\prime}\right) X,\left({ }^{\mathbf{t}} X X^{\prime}-{ }^{\mathbf{t}} X^{\prime} X\right)+\left(U B^{\prime}-U^{\prime} B\right)-{ }^{\mathbf{t}}\left(U B^{\prime}-U^{\prime} B\right)\right)
$$

avec $\widetilde{U}$ la matrice bloc $\left(u_{i j} I_{n}\right)_{1 \leq i, j \leq r}$ pour $U=\left(u_{i j}\right)_{1 \leq i, j \leq r}$, et

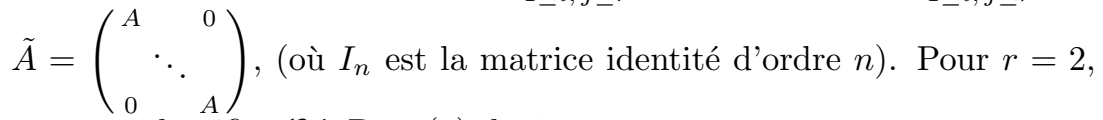
on peut identifier $\mathcal{B}$ à $\mathbb{R}$ et (1) devient

(2) $\quad\left[(U, A, X, b),\left(U^{\prime}, A^{\prime}, X^{\prime}, b^{\prime}\right)\right]=\left(\left[U, U^{\prime}\right],\left[A, A^{\prime}\right],(\widetilde{U}+\tilde{A}) X^{\prime}\right.$

$$
\left.-\left(\widetilde{U}^{\prime}+\tilde{A}^{\prime}\right) X, b^{\prime} \operatorname{trace} U-b \operatorname{trace} U^{\prime}+\left\langle X, X^{\prime}\right\rangle_{J}\right)
$$

$\operatorname{avec}\left\langle X, X^{\prime}\right\rangle_{J}=\left\langle X_{1}, X_{2}^{\prime}\right\rangle-\left\langle X_{2}, X_{1}^{\prime}\right\rangle$ pour $X=\left(X_{1}, X_{2}\right)$ et $X^{\prime}=$ $\left(X_{1}^{\prime}, X_{2}^{\prime}\right)$.

Dans la suite, on considère les sous-algèbres $\mathcal{H}$ de $\mathcal{G}$ qui contiennent $\mathcal{B}$. L'idéal $\mathcal{B}$ n'est pas indécomposable, mais il a la propriété suivante:

Proposition 2.1. Tout sous-espace propre non dégenéré $E$ de $\mathbb{R}^{m}$ invariant par $\mathcal{B}$ est

a) soit contenu dans l'orthogonal de $\Re$ (relativement à \langle\rangle )

b) soit son orthogonal vérifie a).

Autrement dit, on peut supposer que $E=\left\{\left(-{ }^{t} V_{0} Z, Z, 0\right), Z \in F\right\}$ avec $F$ un sous-espace de $\mathbb{R}^{n}$ et $V_{0} \in F^{r}$.

Corollaire 2.2. Toute sous-algèbre qui contient $\mathcal{B}$ a la même propriété ci-dessus. 
Preuve de la proposition: On identifie $\mathbb{R}^{m}$ à $\mathbb{R}^{r} \oplus \mathbb{R}^{n} \oplus \mathbb{R}^{r *}$ avec $\mathbb{R}^{r *}$ le dual de $\mathbb{R}^{r}$ relativement à \langle\rangle . Soit $E$ un sous-espace propre non dégénéré de $\mathbb{R}^{m}$, invariant par $\mathcal{H}$. Soit $V=(T, Z, S) \in E$. Pour $(0,0,0, B) \in \mathcal{B}$, on a:

$$
(0,0,0, B) \cdot V=(B \cdot S, 0,0) \in E .
$$

Supposons qu'il existe $\left(T_{0}, Z_{0}, S_{0}\right) \in E$ avec $S_{0}=\left(t_{1}, \ldots, t_{r}\right) \neq 0$. Alors il existe $\alpha \in\{1, \ldots, r\}$ telle que $t_{\alpha} \neq 0$. Pour $B=\left(b_{i, j}\right)$ avec $b_{1, \alpha}=$ $-b_{\alpha, 1}=1$ et les autres $b_{i, j}=0$, d'après (3), on a $t_{\alpha} e_{1}-t_{1} e_{\alpha} \in E$. Donc il existe $V_{0}^{\prime}=\left(T_{0}^{\prime}, Z_{0}^{\prime}, S_{0}^{\prime}\right) \in E$ isotrope tel que $\left\langle t_{\alpha} e_{1}-t_{1} e_{\alpha}, V_{0}^{\prime}\right\rangle=$ $t_{\alpha} x_{1}-t_{1} x_{\alpha}=1$ avec $S_{0}^{\prime}=\left(x_{1}, \ldots, x_{r}\right)$. Or $x_{\alpha} e_{1}-x_{1} e_{\alpha} \in E$. D'où $e_{1}, e_{r+1-\alpha} \in E$. A nouveau pour $e_{1}$, il existe $V_{1}=\left(T_{1}, Z_{1}, S_{1}\right) \in E$, isotrope tel que $S_{1}=\left(t_{1}, \ldots, t_{r-1}, 1\right)$. En appliquant (3) à $V_{1}$ et avec des choix convenables de $B$, on trouve $\mathbb{R}^{r} \subset E$. D'où la proposition quitte à prendre $E^{\perp}$.

Corollaire 2.3. Si $\mathcal{H}$ est une sous-algèbre de $\mathcal{G}$ qui contient l'idéal $\mathcal{N} \oplus \mathcal{B}$, alors elle est indécomposable.

Preuve: Il suffit de montrer que $\mathcal{N} \oplus \mathcal{B}$ est indécomposable. Soit donc $E$ un sous-espace propre non dégénéré de $\mathbb{R}^{m}$ invariant par $\mathcal{N} \oplus \mathcal{B}$. D'après la proposition, on peut supposer que $E=\left\{\left(-{ }^{t} V_{0} Z, Z, 0\right), Z \in F\right\}$. Pour $(0,0, X, 0) \in \mathcal{N}$ et $\left(-{ }^{t} V_{0} Z, Z, 0\right) \in E$ on a:

$$
(0,0, X, 0) \cdot\left(-{ }^{t} V_{0} Z, Z, 0\right)=\left({ }^{t} X Z, 0,0\right) \in E .
$$

Ce qui implique que ${ }^{t} X Z=0$ pour tout $X \in \mathbb{R}^{r n}$. D'où $E=0$.

Maintenant, on cherche s'il y'a d'autres sous-algèbres $\mathcal{H}$ de $\mathcal{G}$ qui sont indécomposables.

Pour cela, on considère $\mathcal{H}_{1}=\mathcal{H} \cap(\mathcal{N} \oplus \mathcal{B})$. Puisque $\mathcal{B}$ est supposée contenue dans $\mathcal{H}$, on peut écrire $\mathcal{H}_{1}=\mathcal{P} \oplus \mathcal{B}$, où $\mathcal{P}=\mathcal{H} \cap \mathcal{N}$.

Nous supposerons dans toute la suite que $\mathcal{P}$ est inclus strictement dans $\mathcal{N}$. On note $\mathcal{Q}$ l'orthogonal de $\mathcal{P}$ relativement au produit scalaire canonique de $\mathcal{N}$ identifié à $\mathbb{R}^{r n}$. $\mathcal{Q}$ est donc non nul. On note $\mathcal{P}_{i}$ la $i$-ème projection de $\mathcal{P}$ sur $\mathbb{R}^{n}$ (pour $i=1, \ldots, r$ ) et $R$ l'orthogonal de $\mathcal{P}_{1}+\cdots+\mathcal{P}_{r}$ relativement au produit scalaire canonique de $\mathbb{R}^{n}$. Donc $\mathcal{Q}=\mathcal{Q}^{\prime} \oplus R^{r}$ avec $\mathcal{Q}^{\prime}$ l'orthogonal de $R^{r}$ dans $\mathcal{Q}$, relativement au produit scalaire canonique de $\mathcal{N}$.

Proposition 2.4. Soit $\mathcal{H}$ une sous-algèbre de $\mathcal{G}$ qui contient $\mathcal{B}$. Alors si $\mathcal{P}_{1}+\cdots+\mathcal{P}_{r}=\mathbb{R}^{n}, \mathcal{H}$ est indécomposable. Et si $\mathcal{H}_{1}=\mathcal{H}$, c'est à dire $\mathcal{H} \subset \mathcal{B} \oplus \mathcal{N}$ cette condition est nécessaire et suffisante. 
Preuve: Soit $E=\left\{\left(-{ }^{t} V_{0} Z, Z, 0\right), Z \in F\right\}$ un sous-espace propre non dégénéré de $\mathbb{R}^{m}$ invariant par $\mathcal{H}$, avec $F$ un sous-espace non nul de $\mathbb{R}^{n}$ et $V_{0} \in F^{r}$. Pour $(0,0, X, 0) \in \mathcal{P}$, on a:

$$
(0,0, X, 0) \cdot\left(-{ }^{t} V_{0} \cdot Z, Z, 0\right)=\left({ }^{t} X \cdot Z, 0,0\right) \in E .
$$

Ce qui implique que ${ }^{t} X_{i} \cdot Z=0$ pour $X=\left(X_{1}, \ldots, X_{r}\right)$. C'est à dire $Z$ est orthogonal à $\mathcal{P}_{i}$, donc aussi à $\mathcal{P}_{1}+\cdots+\mathcal{P}_{r}$. Par suite $E=0$.

Maintenant, si $\mathcal{H} \subset \mathcal{B} \oplus \mathcal{N}$ et si $\mathcal{H}$ est indécomposable, alors nécessairement $\mathcal{P}_{1}+\cdots+\mathcal{P}_{r}=\mathbb{R}^{n}$. Sinon $\left\{(0, Z, 0), Z \in\left(\mathcal{P}_{1}+\cdots+\mathcal{P}_{r}\right)^{\perp}\right\}$ est un sous-espace propre non dégénéré de $\mathbb{R}^{m} \mathcal{H}$-invariant.

Nous supposons désormais que l'espace $\mathcal{P}_{1}+\cdots+\mathcal{P}_{r}$ est inclus strictement dans $\mathbb{R}^{n}$ et on note $R$ l'orthogonal de $\mathcal{P}_{1}+\cdots+\mathcal{P}_{r}$ dans $\mathbb{R}^{n}$ relativement au produit scalaire ordinaire. Pour chercher d'autres possibilités de $\mathcal{H}$, on a besoin de certaines notations.

Notations. On note $\mathcal{U}^{\prime}$ (resp. $\mathcal{K}^{\prime}$ ) la projection de $\mathcal{H}$ sur $\mathcal{U}$ (resp. sur $\mathcal{K})$.

On pose $\mathcal{D}=\left\{A \in \mathcal{K}^{\prime}\right.$ tel que $\left.(0, A, X, 0) \in \mathcal{H}\right\}$, et on note $\mathcal{K}_{1}$ (resp. $\left.\mathcal{K}_{2}\right)$ la sous-algèbre de $\mathcal{K}$ qui laisse invariant $R^{\perp}$ (resp. $R$ ) et qui annule $R$ (resp. $R^{\perp}$ ). On note $\mathcal{D}_{2}$ la projection de $\mathcal{D}$ sur $\mathcal{K}_{2}$. On note aussi $\mathcal{D}^{\prime}$ le supplémentaire orthogonal de $\mathcal{D}$ dans $\mathcal{K}^{\prime}$ relativement à la forme de Killing de $\mathcal{K}$. Par définition, il existe une application linéaire $\varphi: \mathcal{U}^{\prime} \rightarrow$ $\mathcal{D}^{\prime}$.

On note $\mathcal{L}=\{(U, A, X, 0) \in \mathcal{H} / X \in \mathcal{Q}\}$ et $\mathcal{L}_{1}=\mathcal{U}^{\prime} \oplus \mathcal{K}^{\prime}$. Par définition, il existe deux applications linéaires $\psi: \mathcal{L}_{1} \rightarrow \mathcal{Q}^{\prime}$ et $\phi: \mathcal{L}_{1} \rightarrow R^{r}$ telles que si on pose $\omega=\psi+\phi, \mathcal{L}=\{(U, A, \omega(U, A), 0) \in \mathcal{H}$ tel que $\left.(U, A) \in \mathcal{L}_{1}\right\}$. Clairement, $\mathcal{H}=\mathcal{L} \oplus \mathcal{P} \oplus \mathcal{B}$ et $\mathcal{L}=\mathcal{L}^{\prime} \oplus \mathcal{L}_{D}$ avec $\mathcal{L}^{\prime}=\left\{(U, \varphi(U), \omega(U, \varphi(U)), 0) \in \mathcal{H}\right.$ tel que $\left.U \in \mathcal{U}^{\prime}\right\}$ et $\mathcal{L}_{D}=$ $\{(0, A, \omega(0, A), 0) \in \mathcal{H}$ tel que $A \in \mathcal{D}\}$.

Une première condition nécessaire pour que $\mathcal{H}$ soit indécomposable est la suivante:

Proposition 2.5. Si $\mathcal{H}$ est indécomposable, alors tout élément non nul du centre de $\mathcal{U}^{\prime}$ admet au moins une valeur propre imaginaire pure ou nulle.

\section{Preuve:}

Lemme 2.6. Si $\mathcal{K}^{\prime}$ laisse invariant un sous-espace $F \neq 0$ de $R$ et s'il existe un $V_{0} \in F^{r}$ tels que $\phi_{F}(U, A)=(\widetilde{U}+\widetilde{A})_{F} \cdot V_{0}$, pour tout $(U, A) \in \mathcal{L}_{1}$ avec $\phi_{F}(U, A)$ la projection de $\phi(U, A)$ sur $F^{r}$ et $(\widetilde{U}+\widetilde{A})_{F}$ la restriction de $(\widetilde{U}+\widetilde{A}) \grave{a} F^{r}$. 
Alors le sous-espace propre $E=\left\{\left(-{ }^{t} V_{0} Z, Z, 0\right) / Z \in F\right\}$ est $\mathcal{H}$-invariant et par suite $\mathcal{H}$ est décomposable.

Preuve du Lemme 2.6: L'image de $E$ par $\mathcal{P} \oplus \mathcal{B}$ est nulle. En plus,

$$
\begin{aligned}
(U, A, \omega(U, A), 0) \cdot\left(-{ }^{t} V_{0} Z, Z, 0\right) & =\left(U\left(-{ }^{t} V_{0} Z\right)+{ }^{t} \omega(U, A) \cdot Z, A Z, 0\right) \\
& =\left(U\left(-{ }^{t} V_{0} Z\right)+{ }^{t} \phi_{F}(U, A) \cdot Z, A Z, 0\right) \\
& =\left({ }^{t}\left(-\widetilde{U} V_{0}+(\widetilde{U}+\widetilde{A}) V_{0}\right) Z, A Z, 0\right) \\
& =\left(-{ }^{t} V_{0}(A Z), A Z, 0\right) .
\end{aligned}
$$

Fin de la preuve de la Proposition 2.5: Supposons que le centre $\mathcal{C}\left(\mathcal{U}^{\prime}\right)$ de $\mathcal{U}^{\prime}$ contient un élément $U_{0}$ dont les parties réelles de ses valeurs propres sont toutes non nulles. Soit $\left(U_{0}, A_{0}, X_{0}\right) \in \mathcal{H}$ avec $X_{0} \in \mathcal{Q}$. $\widetilde{U_{0}}$ commute avec $\widetilde{A_{0}}$, en plus $A_{0}$ et $\widetilde{A_{0}}$ sont anti-symétriques. Donc si on note $P_{a d A_{0}}$ le polynôme caractéristique de $a d A_{0}$, alors $\widetilde{U_{0}}+\widetilde{A_{0}}$ et $P_{a d A_{0}}\left(\widetilde{U_{0}}+\widetilde{A_{0}}\right)$ sont deux matrices inversibles. D'après $(1)$, pour $(U, A)$ et $\left(U^{\prime}, A^{\prime}\right) \in \mathcal{L}_{1}$, $\phi$ satisfait:

$$
\phi\left(\left[U, U^{\prime}\right],\left[A, A^{\prime}\right]\right)=(\widetilde{U}+\widetilde{A}) \phi\left(U^{\prime}, A^{\prime}\right)-\left(\widetilde{U^{\prime}}+\widetilde{A^{\prime}}\right) \phi(U, A) .
$$

D'où

$$
\phi\left(0, a d A_{0} \cdot A\right)=\left(\widetilde{U_{0}}+\widetilde{A_{0}}\right) \phi(U, A)-(\widetilde{U}+\widetilde{A}) X_{1},
$$

avec $X_{1}$ la projection de $X_{0}$ sur $R^{r}$.

On pose $\theta(U, A)=\phi(U, A)-(\widetilde{U}+\widetilde{A}) X_{2}$, avec $\left.X_{2}=\widetilde{\left(U_{0}\right.}+\widetilde{A_{0}}\right)^{-1} X_{1}$. De $(5)$, on déduit $\theta\left(0, a d^{m} A_{0} \cdot A\right)=\left(\widetilde{U_{0}}+\widetilde{A_{0}}\right)^{m} \cdot \theta(U, A)$, pour tout $m$ entier positif.

Ce qui implique

$$
\theta\left(0, P_{a d A_{0}} \cdot\left(a d A_{0}\right) \cdot A\right)=P_{a d A_{0}}\left(\widetilde{U_{0}}+\widetilde{A_{0}}\right) \theta(U, A)
$$

donc $\theta(U, A)=0$. Ainsi $\phi(U, A)=(\widetilde{U}+\widetilde{A}) X_{1}$. D'après le Lemme 2.6, $\mathcal{H}$ est donc décomposable. Contradiction.

Une deuxième condition nécessaire pour que $\mathcal{H}$ soit indécomposable est la suivante:

Proposition 2.7. Si $\mathcal{H}$ est indécomposable, alors nécessairement $\mathcal{D}_{2}=0$. 
Preuve:

Lemme 2.8 (Premier lemme de Whitehead). Soit $\mathbf{K}$ une algèbre de Lie semi-simple, alors le groupe de cohomologie $H^{1}(\mathbf{K})$ de $\mathbf{K}$ est nul.

Lemme 2.9 (Corollaire du Lemme 2.8). Soit $\mathbf{K} \neq 0$ une sousalgèbre de $\operatorname{so}(q, \mathbb{R})$. On note $F_{1}$ l'orthogonal de l'intersection des noyaux des éléments de $\mathbf{K}$ et $\mathbf{K}_{2}$ la restriction de $\mathbf{K}$ à $F_{1}$. Alors

a) L'élément de Casimir $\mathbf{c}$ de $\mathbf{K}$ commute avec tout élément de $\mathbf{K}$. En plus $\mathbf{c}=\sum_{i} D_{i}^{2}$ avec $\left(D_{i}\right)$ une base orthonormale de $\mathbf{K}$ relativement à la forme de Killing de $\operatorname{so}(q, \mathbb{R})$.

b) Si $\mathbf{K}$ est semi-simple ou commutative, l'élément de Casimir de $\mathbf{K}_{2}$ est inversible.

c) Si $\theta: \mathbf{K} \rightarrow \mathbb{R}^{q}$ est un cocycle: $\theta([A, B])=A \theta(B)-B \theta(A)$ pour tout $A, B \in \mathbf{K}$, alors c'est un cobord: il existe $V_{0} \in F_{1}$ tel que $\theta_{1}(A)=A \cdot V_{0}$ avec $\theta_{1}(A)$ la projection de $\theta(A)$ sur $F_{1}$.

Preuve de la Proposition 2.7: On suppose maintenant que $\mathcal{D}_{2} \neq 0$. De (1), pour $A \in \mathcal{D}$ et $U \in \mathcal{U}^{\prime}$ on a:

$$
(0,[A, \varphi(U)], \widetilde{A} \omega(U, \varphi(U))-(\widetilde{U}+\widetilde{\varphi(U)}) \omega(0, A), 0) \in \mathcal{H}
$$

Ce qui implique que $\mathcal{D}$ est un idéal de $\mathcal{K}^{\prime}$ et donc $\mathcal{D}^{\prime}$ aussi. Par suite $\varphi$ et $\omega$ satisfaient

$$
[A, \varphi(U)]=0
$$

et

$$
\widetilde{A} \omega(U, \varphi(U))-(\widetilde{U}+\widetilde{\varphi(U)}) \omega(0, A) \in \mathcal{P} .
$$

Or $R^{r}$ est invariant par $\widetilde{A}$ et $(\widetilde{U}+\widetilde{\varphi(U)})$, donc

$$
\widetilde{A} \phi(U, \varphi(U))=(\widetilde{U}+\widetilde{\varphi(U)}) \phi(0, A) .
$$

De (1), $\omega$ satisfait aussi:

$$
\omega(0,[A, B])-(\widetilde{A} \omega(0, B)-\widetilde{B} \omega(0, A)) \in \mathcal{P}, \text { pour } A, B \in \mathcal{D} .
$$

Ce qui implique

$$
\phi(0,[A, B])=\widetilde{A} \phi(0, B)-\widetilde{B} \phi(0, A)
$$


et donc pour chaque $i=1, \ldots, r$

$$
\phi_{i}(0,[A, B])=A \phi_{i}(0, B)-B \phi_{i}(0, A)
$$

On pose $\mathcal{C}_{1}=\mathcal{D} \cap \mathcal{K}_{1}$ et on note $\mathcal{C}_{2}$ l'orthogonal de $\mathcal{C}_{1}$ dans $\mathcal{K}^{\prime}$ relativement à la forme de Killing de $\mathcal{K}$. $\mathcal{C}_{1}$ et $\mathcal{C}_{2}$ sont deux idéaux de $\mathcal{D}$. $\mathcal{D}_{2}$ est la projection de $\mathcal{C}_{2}$ sur $\mathcal{K}_{2}$. En plus la projection $p: \mathcal{C}_{2} \rightarrow \mathcal{D}_{2}$ est un isomorphisme d'algèbre de Lie. On pose $F_{0}$ l'intersection des noyaux des éléments de $\mathcal{D}_{2}$, et $F_{1}$ l'orthogonal de $F_{0}$ dans $R$. Pour $A \in \mathcal{C}_{2}$, on pose $A_{2}=p(A)$ qu'on peut identifier à sa restriction à $F_{1}$. $\mathcal{D}_{2}$ est supposé $\neq 0$, donc $F_{1} \neq 0$. Si on pose $\theta_{i}\left(A_{2}\right)=\phi_{i}(0, A)$, (7) implique, pour $A_{2}, A_{2}^{\prime} \in \mathcal{D}_{2}$ :

$$
\theta_{i}\left(\left[A_{2}, A_{2}^{\prime}\right]\right)=A_{2} \theta_{i}\left(A_{2}^{\prime}\right)-A_{2}^{\prime} \theta_{i}\left(A_{2}\right) \text {. }
$$

D'après le Lemme 2.8, il existe $V_{i} \in F_{1}$ tel que pour $A \in \mathcal{C}_{2}, \theta_{i, F_{1}}(A)=$ $A \cdot V_{i}$, avec $\theta_{i, F_{1}}(A)$ la projection de $\theta_{i}(A)$ sur $F_{1}$. D'où $\phi_{F_{1}}(0, A)=\widetilde{A} V_{0}$ avec $V_{0}=\left(V_{1}, \ldots, V_{r}\right) \in F_{1}^{r}$. De (6), on déduit: pour $U \in \mathcal{U}^{\prime}$,

$$
\widetilde{A_{2}}\left(\phi_{F_{1}}(U, \varphi(U))-(\widetilde{U}+\widetilde{\varphi(U)}) V_{0}\right)=0
$$

Avec $\phi_{F_{1}}(U, \varphi(U))$ la projection de $\phi(U, \varphi(U))$ sur $F_{1}^{r}$. Du Lemme 2.9, on déduit $\phi_{F_{1}}(U, \varphi(U))=(\widetilde{U}+\widetilde{\varphi(U)}) V_{0}$. Puisque $\mathcal{C}_{1}$ et $\mathcal{C}_{2}$ sont deux idéaux de $\mathcal{D}$, de $(7)$, on obtient $\widetilde{A} \phi(0, B)=0$, pour $A \in \mathcal{C}_{2}$ et $B \in \mathcal{C}_{1}$. Autrement dit, $\phi(0, B) \in F_{0}^{r}$. En vertu du Lemme 2.6, $\mathcal{H}$ est donc décomposable. Contradiction.

Remarque. $\mathcal{K} \oplus \mathcal{N} \oplus \mathcal{B}$ est un idéal de $\mathcal{G}$, donc $\mathcal{H} \cap(\mathcal{K} \oplus \mathcal{N} \oplus \mathcal{B})$ est un idéal de $\mathcal{H}$.

De la Proposition 2.7 on déduit immédiatement

Corollaire 2.10. Si on note $\phi_{i}$ les composantes de l'application $\phi$, alors si $R=\phi_{1}(\mathcal{D})+\cdots+\phi_{r}(\mathcal{D}), \mathcal{H}$ est indécomposable et cette condition est nécessaire et suffisante si $\mathcal{L}_{D}=\mathcal{H}$. C'est à dire $\mathcal{H} \subset \mathcal{K} \oplus \mathcal{N} \oplus \mathcal{B}$.

Nous supposons désormais que l'espace $\phi_{1}(\mathcal{D})+\cdots+\phi_{r}(\mathcal{D})$ est inclus strictement dans $R$ et on note $R^{\prime}$ son orthogonal dans $R$. Une autre condition nécessaire pour que $\mathcal{H}$ soit indécomposable est la suivante:

Proposition 2.11. Si $R^{\prime} \neq 0$ et si $\mathcal{H}$ est indécomposable, alors $\mathcal{U}^{\prime}$ n'est pas semi-simple. 
Preuve: Supposons que $\mathcal{U}^{\prime}$ est semi-simple. Soit $\varphi_{2}: \mathcal{U}^{\prime} \rightarrow \mathcal{K}_{2}$ la projection de $\varphi$ sur $\mathcal{K}_{2}$. $\varphi_{2}$ est une représentation. Donc $\varphi_{2}\left(\mathcal{U}^{\prime}\right)$ est semi-simple ou nulle. On note $\widetilde{U_{R}^{\prime}}$ l'ensemble des matrices bloc $\widetilde{U_{R}}=$ $\left(u_{i j} I_{R}\right)_{1 \leq i, j \leq r}$ avec $U=\left(u_{i j}\right)_{1 \leq i, j \leq r} \in \mathcal{U}^{\prime}$ et $I_{R}$ la matrice identité de $R$. On pose $\mathcal{V}=\widetilde{U_{R}^{\prime}} \oplus \varphi_{2}\left(\mathcal{U}^{\prime}\right)$. Puisque $\mathcal{U}^{\prime}$ est semi-simple alors $\mathcal{V}$ l'est. On définit $\xi: \mathcal{V} \rightarrow R^{r}$ par $\xi(W)=\phi(U, \varphi(U))$ pour $W=\widetilde{U_{R}}+\widetilde{\varphi_{2}(U)}$. Pour $W^{\prime}=\widetilde{U_{R}^{\prime}}+\widetilde{\varphi_{2}\left(U^{\prime}\right)}$, on a: $\left.\left.\left[W, W^{\prime}\right]=\widetilde{\left[U, U^{\prime}\right.}\right]_{R}+\widetilde{\varphi_{2}\left(\left[U^{\prime} U^{\prime}\right]\right.}\right)$, donc de (1), $\xi$ satisfait

$$
\xi\left(\left[W, W^{\prime}\right]\right)=W \xi\left(W^{\prime}\right)-W^{\prime} \xi(W) .
$$

D'après le Lemme 2.8, il existe $X_{0} \in R^{r}$ telle que $\xi(W)=W \cdot X_{0}$. Soit $\phi(U, \varphi(U))=\left(\widetilde{U_{R}}+\widetilde{\varphi_{2}(U)}\right) \cdot X_{0}$. Et d'après (6) et la Proposition 2.7, $\left(\widetilde{U_{R}}+\widetilde{\varphi_{2}(U)}\right) \cdot \phi(0, A)=0$, pour $A \in \mathcal{D}$. Ce qui implique que $R^{\prime}$ est invariant par $\varphi_{2}\left(\mathcal{U}^{\prime}\right)$. Par conséquent, le sous espace-propre $\left\{\left(-{ }^{t} X_{0} Z, Z, 0\right) / Z \in R^{\prime}\right\}$ est $\mathcal{H}$-invariant. Absurde.

\section{Cas de signature $(2, n+2)$}

Maintenant, on se restreint au cas de type $(2, n+2)(r=2)$ et on cherche les autres possibilités de $\mathcal{H}$ contenant l'idéal $\mathcal{B}$. On suppose toujours que $R^{\prime} \neq 0$. En vertu des Propositions 2.5 et 2.11, on obtient

Proposition 3.12. A une conjuguaison près dans $g l(2, \mathbb{R}), \mathcal{U}^{\prime}$ ne peut être que l'une des cas suivants:

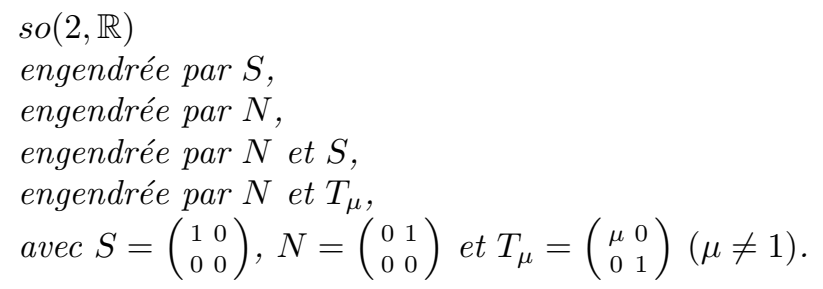

Preuve facile.

Attention. A une conjuguaison près, la sous-algèbre engendrée par $N$ et $S$ et la sous-algèbre engendrée par $N$ et $T_{0}$ sont égales. Mais vues comme traces respectivement de deux sous-algèbres $\mathcal{H}_{1}$ et $\mathcal{H}_{2}$ de $\mathcal{H}$, elles sont différentes. En effet $\mathcal{H}_{2}$ annule une droite isotrope, alors que ce n'est pas le cas pour $\mathcal{H}_{1}$.

On regarde maintenant, pour chaque cas de la Proposition 3.12, quelles sont les conditions nécessaires et suffisantes pour que $\mathcal{H}$ soit indécomposable. 
3.0.1. Cas où $\mathcal{U}^{\prime}=s o(2, \mathbb{R})$.

Dans ce cas $\mathcal{L}^{\prime}$ est engendrée par $\left(J, A_{0}, X_{0}, 0\right)$ avec $A_{0}=\varphi(J), X_{0}=$ $\omega(J, \varphi(J))=Y_{0}+Z_{0} \in \mathcal{Q}^{\prime} \oplus R^{2}$ et $J=\left(\begin{array}{cc}0 & 1 \\ -1 & 0\end{array}\right)$. A une conjuguaison près, on peut supposer que

$$
A_{0}=\left(\begin{array}{ccc}
\lambda_{1} J & & 0 \\
& \ddots & \\
0 & & \lambda_{s} J
\end{array}\right) \quad \text { avec } \quad \lambda_{k} \in \mathbb{R}, k=1, \ldots, s
$$

Si on note $A_{2}$ la restriction de $A_{0}$ à $R^{\prime}$ et $S p\left(A_{2}\right)$ le spectre de $A_{2}$, on a

Proposition 3.13. $\mathcal{H}$ est indécomposable, si et seulement si, $S p\left(A_{2}\right)=\{-i,+i\}$ et $A_{2} Z_{1, F} \neq-Z_{2, F} \neq 0$ pour tout sous espace $F$ de $R^{\prime}$ invariant par $A_{2}$. Avec $i$ l'imaginaire pure et $Z_{0}=\left(Z_{1}, Z_{2}\right)$, où $Z_{j, F}$ est la projection de $Z_{j}$ sur $F$, pour $j=1,2$.

Preuve: On suppose que $S p\left(A_{2}\right) \neq\{-i,+i\}$, donc il existe $\lambda \neq \pm 1 \in \mathbb{R}$ telle que $\pm \lambda i \in S p\left(A_{2}\right)$. Soit donc $X_{1}$ et $X_{2}$ deux vecteurs indépendantes de $R^{\prime}$ tels que $A_{2} X_{1}=-\lambda X_{2}$ et $A_{2} X_{2}=\lambda X_{1}$. Si on note $F_{0}$ le sousespace engendré par $X_{1}$ et $X_{2}$ alors $\left(\widetilde{J}+\widetilde{A_{0}}\right)_{F_{0}}$ est inversible. D'après le Lemme 2.6, on en déduit que $\mathcal{H}$ est décomposable. Par suite $\operatorname{Sp}\left(A_{2}\right)=$ $\{-i,+i\}$. Si maintenant il existe un sous-espace $F_{0} \neq 0$ de $R^{\prime}$ invariant par $A_{2}$ telle que $\left(\widetilde{J}-\widetilde{A_{0}}\right) Z_{0, F_{0}}=0$, alors

$$
\begin{aligned}
& \left(\widetilde{J}+\widetilde{A_{0}}\right) V_{0}=Z_{0, F_{0}}, \text { avec } V_{0}=\left(-Z_{2, F_{0}}, 0\right) \\
& \text { et } Z_{2, F_{0}} \text { la deuxième composante de } Z_{0, F_{0}} .
\end{aligned}
$$

Inversement, si $\mathcal{H}$ laisse invariant un sous-espace propre $E=\left\{\left(-{ }^{t} X_{1} Z, Z, 0\right) / Z \in F_{0} \subset R^{\prime}\right\}$, on a:

$$
\begin{aligned}
\left(J, A_{0}, X_{0}, 0\right)^{2} \cdot & \left(-{ }^{t} X_{1} Z, Z, 0\right) \\
& =\left({ }^{t} X_{1} Z+J\left({ }^{t} Z_{0, F_{0}} \cdot Z\right)+{ }^{t} Z_{0, F_{0}} \cdot A_{2} Z,-Z, 0\right) \in E .
\end{aligned}
$$

Ce qui implique $\left(\widetilde{J}-\widetilde{A_{0}}\right) Z_{0, F_{0}}=0$. Impossible.

\subsubsection{Cas où $\mathcal{U}^{\prime}$ est engendrée par $N$.}

$\mathcal{L}^{\prime}$ est engendrée par $\left(N, A_{N}, X_{N}, 0\right)$ avec $A_{N}=\varphi(N), X_{N}=$ $\omega(N, \varphi(N))=Y_{N}+Z_{N} \in \mathcal{Q}^{\prime} \oplus R^{2}$. 
Proposition 3.14. Si on note $A_{N}^{\prime}$ la restriction de $A_{N} \grave{a} R^{\prime}, Z_{N}^{\prime}$ la projection de $Z_{N}$ sur $R^{\prime 2}$ et $Z_{N, 2}^{\prime}$ la deuxième composante de $Z_{N}^{\prime}$, alors $A_{N}^{\prime}=0$. Et $\mathcal{H}$ est indécomposable si et seulement si $R^{\prime}=\operatorname{vect}\left\{Z_{N, 2}^{\prime}\right\}$.

Preuve: Supposons que $A_{N}^{\prime} \neq 0$. Donc si on note $F$ l'orthogonal du noyau de $A_{N}^{\prime}$ dans $R^{\prime}$, la restriction de $A_{N}^{\prime}$ à $F^{2}$ est inversible et $\left(\widetilde{N}+\widehat{A}_{N}\right)_{F}$ aussi. Impossible. D'où $A_{N}^{\prime}=0$. Maintenant, si l'orthogonal $R^{\prime \prime}$ de vect $\left\{Z_{N, 2}^{\prime}\right\}$ dans $R^{\prime}$ est $\neq 0$, alors $\mathcal{H}$ laisse invariant le sous-espace propre $\left\{\left(-{ }^{t} V_{0} Z, Z, 0\right), Z \in R^{\prime \prime}\right\}$ avec $V_{0}=\left(0,-Z_{N, 1}\right)\left(Z_{N, 1}\right.$ la premième composante de $\left.Z_{N}\right)$. Inversement, si $\mathcal{H}$ laisse invariant un sous-espace propre $E=\left\{\left(-{ }^{t} X_{1} Z, Z, 0\right) / Z \in F\right\}$, alors $F \subset R^{\prime}$ et $F \subset\left\{Z_{N, 2}^{\prime}\right\}^{\perp}$. D'où $F=0$.

\subsubsection{Cas où $\mathcal{U}^{\prime}$ est engendrée par $S$.}

$\mathcal{L}^{\prime}$ est engendrée par $\left(S, A_{S}, X_{S}, 0\right)$ avec $A_{S}=\varphi(S), \quad X_{S}=$ $\omega(S, \varphi(S))=Y_{S}+Z_{S} \in \mathcal{Q}^{\prime} \oplus R^{2}$. De (1), pour $A \in \mathcal{D}$, on a:

$$
\phi\left(\left[A_{S}, A\right]\right)=\left(\widetilde{S}+\widetilde{A_{S}}\right) \phi(A) .
$$

Donc

$$
\phi_{1}\left(\left[A_{S}, A\right]\right)=\left(I+A_{S}\right) \cdot \phi_{1}(A) \text { et } \phi_{2}\left(\left[A_{S}, A\right]\right)=A_{S} \cdot \phi_{2}(A) .
$$

Ce qui implique $\phi_{1}(A)=0$. Avec la même démonstration de la Proposition 3.14 , on obtient

Proposition 3.15. $\phi_{1}(\mathcal{D})=0, A_{S}^{\prime}=0$ et $\mathcal{H}$ est indécomposable, si et seulement si, $R^{\prime}=\operatorname{vect}\left\{Z_{S, 2}^{\prime}\right\}$. Avec $A_{S}^{\prime}$ la restriction de $A_{S}$ à $R^{\prime}$, $Z_{S}^{\prime}$ la projection de $Z_{S}$ sur $R^{\prime 2}$ et $Z_{S, 2}^{\prime}$ la deuxième composante de $Z_{S}^{\prime}$.

\subsubsection{Cas où $\mathcal{U}^{\prime}$ est engendrée par $N$ et $S$.}

$\mathcal{L}^{\prime}$ est engendrée par $\left(N, A_{N}, X_{N}, 0\right)$ et $\left(S, A_{S}, X_{S}, 0\right)$. Pour $A \in \mathcal{D}, \phi$ vérifie:

$$
\left\{\begin{array}{l}
\phi_{1}(A)=0 \\
\phi\left(\left[A_{N}, A\right]\right)=\left(\widetilde{N}+\widetilde{A_{N}}\right) \phi(A) .
\end{array}\right.
$$

Ce qui implique

$$
\phi(\mathcal{D})=0
$$

Autrement dit, $R=R^{\prime}$. On a aussi

$$
\left(N,\left[A_{S}, A_{N}\right],\left(\widetilde{S}+\widetilde{A_{S}}\right) X_{N}-\left(\widetilde{N}+\widetilde{A_{N}}\right) X_{S}, 0\right) \in \mathcal{H}
$$


D'où

$$
\left(0,\left[A_{S}, A_{N}\right]-A_{N},\left(\widetilde{S}+\widetilde{A_{S}}-I\right) X_{N}-\left(\widetilde{N}+\widetilde{A_{N}}\right) X_{S}, 0\right) \in \mathcal{H}
$$

Puisque $\mathcal{D}_{2}=0$, de $(8)$ on a immédiatement $\left[A_{S}^{\prime}, A_{N}^{\prime}\right]=A_{N}^{\prime}$ et $\left(\widetilde{S}+\widetilde{A_{S}^{\prime}}-I\right) Z_{N}=\left(\widetilde{N}+\widetilde{A_{N}^{\prime}}\right) Z_{S}$. Or $A_{S}^{\prime}$ est antisymétrique, donc 0 est la seule valeur propre réelle de $a d A_{S}^{\prime}$. Par suite

$$
A_{N}^{\prime}=0 \text { et }\left(\widetilde{S}+\widetilde{A_{S}^{\prime}}-I\right) Z_{N}=\widetilde{N} \cdot Z_{S}
$$

Puisque $\widetilde{N}$ et $\widetilde{A_{S}^{\prime}}$ commutent on a aussi

$$
\left(\widetilde{S}+\widetilde{A_{S}^{\prime}}-I\right) \widetilde{N}=\widetilde{N}\left(\widetilde{S}+\widetilde{A_{S}^{\prime}}\right)
$$

Maintenant, on suppose que $A_{S}^{\prime} \neq 0$, donc si on note $F$ l'orthogonal du noyau de $A_{S}^{\prime}$ dans $R$, la restriction de $A_{S}^{\prime}$ à $F^{2}$ est inversible et $\left(\widetilde{S}+\widetilde{A_{S}}\right)_{F}$ aussi. Soit $V_{0} \in F^{2}$ tel que $\left(\widetilde{S}+\widetilde{A_{S}}\right)_{F} V_{0}=Z_{S, F}$. Or $F^{2}$ est invariant par $\widetilde{S}, \widetilde{N}$ et $\widetilde{A_{S}^{\prime}}$, donc de (9) et (10) on en déduit $\left(\widetilde{S}+\widetilde{A_{S}^{\prime}}-I\right)_{F} \cdot\left(\widetilde{N} \cdot V_{0}-Z_{N, F}\right)=0 .\left(\widetilde{S}+\widetilde{A_{S}^{\prime}}-I\right)_{F}$ est inversible, par suite $\widetilde{N} \cdot V_{0}=Z_{N, F}$. Impossible. D'où

Proposition 3.16. $\phi(\mathcal{D})=0$ et $A_{N}^{\prime}=A_{S}^{\prime}=0$. En plus $\mathcal{H}$ est indécomposable, si et seulement si, $R^{\prime}=R=\operatorname{vect}\left\{Z_{N, 2}, Z_{S, 2}\right\}$.

Preuve: Supposons que $R=\operatorname{vect}\left\{Z_{N, 2}, Z_{S, 2}\right\}$. Si $\mathcal{H}$ laisse invariant un sous-espace propre $E=\left\{\left(-{ }^{t} X_{0} Z, Z, 0\right) / Z \in F \subset R\right\}$ alors,

$$
\left(N, A_{N}, X_{N}, 0\right) \cdot\left(-{ }^{t} X_{0} Z, Z, 0\right)=\left({ }^{t}\left(Z_{N}-\widetilde{N} \cdot X_{0}\right) Z,-Z, 0\right) \in E .
$$

D'où ${ }^{t}\left(Z_{N}-\widetilde{N} \cdot X_{0}\right) Z=0$. On a de même ${ }^{t}\left(Z_{S}-\widetilde{S} \cdot X_{0}\right) Z=0$. Ce qui implique que $Z$ est orthogonal à $Z_{N, 2}$ et $Z_{S, 2}$. Inversement, si on pose $F_{0}=\left\{Z_{N, 2}, Z_{S, 2}\right\}^{\perp} \cap R \neq 0,\left\{\left(-^{t}\left(Z_{S, 2}, Z_{N, 2}\right) Z, Z, 0\right) / Z \in F_{0}\right\}$ est $\mathcal{H}$-invariant.

3.0.5. Cas où $\mathcal{U}^{\prime}$ est engendrée par $N$ et $T_{\mu}$.

$\mathcal{L}^{\prime}$ est engendrée par $\left(N, A_{N}, X_{N}, 0\right)$ et $\left(T_{\mu}, A_{T}, X_{T}, 0\right)$ avec $A_{T}=$ $\varphi\left(T_{\mu}\right), X_{T}=\omega\left(T_{\mu}, \varphi\left(T_{\mu}\right)\right)=Y_{T}+Z_{T} \in \mathcal{Q}^{\prime} \oplus R^{2}$. Si on pose $\lambda=\mu-1 \neq$ 0 , on a:

(11) $\left(0,\left[A_{T}, A_{N}\right]-\lambda A_{N},\left(\widetilde{T_{\mu}}+\widetilde{A_{T}}-\lambda I\right) X_{N}-\left(\widetilde{N}+\widetilde{A_{N}}\right) X_{T}, 0\right) \in \mathcal{H}$. 
Ce qui implique $\left[A_{T}^{\prime}, A_{N}^{\prime}\right]=\lambda A_{N}^{\prime}$ avec $A_{T}^{\prime}$ la restriction de $A_{T}$ à $R$. D'où $A_{N}^{\prime}=0$. On a aussi pour $A \in \mathcal{D}, \phi_{2}(A)=0, \phi_{1}\left(\left[A_{N}, A\right]\right)=$ $A_{N} \cdot \phi(A), \phi_{1}\left(\left[A_{T}, A\right]\right)=\left(\mu I+A_{T}\right) \phi_{1}(A)$ et $\phi_{2}\left(\left[A_{T}, A\right]\right)=\left(I+A_{T}\right) \phi_{2}(A)$. Maintenant nous distinguerons deux cas:

(a) Si $\mu \neq 0$.

On a donc $\phi(\mathcal{D})=0$ et $\left(\widetilde{T_{\mu}}+\widetilde{A_{T}^{\prime}}-\lambda I\right) Z_{N}=\widetilde{N} \cdot Z_{T}$. En plus $\left(\widetilde{T_{\mu}}+\widetilde{A_{T}^{\prime}}-\lambda I\right) \widetilde{N}=\widetilde{N}\left(\widetilde{T_{\mu}}+\widetilde{A_{T}^{\prime}}\right)$. D'où $\left(I+A_{T}^{\prime}\right) Z_{N, 1}=Z_{N, 2}$ et $\left((2-\mu) I+A_{N}^{\prime}\right) Z_{N, 2}=0$. Avec la même démonstration de la Proposition 3.16 on obtient

Proposition 3.17. Si $\mu \neq 0$, alors $A_{N}^{\prime}=A_{T}^{\prime}=0, \phi(\mathcal{D})=0, Z_{N, 1}=$ $Z_{N, 2}$ et $Z_{N, 2}=0$ si $\mu=2$. En plus $\mathcal{H}$ est indécomposable, si et seulement si, $R^{\prime}=R=\operatorname{vect}\left\{Z_{N, 2}\right\}$.

(b) Si $\mu=0$.

Puisque $\phi_{2}(A)=0$, alors la deuxième composante de $\left(\widetilde{T}+\widetilde{A_{T}}-\right.$ $\lambda I) Z_{N}-\tilde{N} \cdot Z_{T}$ est nulle $\left(T=T_{0}\right)$, d'où $Z_{N, 2}=0$. Supposons maintenant que $\mathcal{H}$ laisse invariant un sous-espace propre $E=\left\{\left(-{ }^{t} V_{0} Z, Z, 0\right) / Z \in F\right\}$ alors $F \subset R^{\prime}$ et

$$
\left\{\begin{array}{l}
{ }^{t}\left(\left(\widetilde{T}+\widetilde{A_{T}}\right) V_{0}-Z_{T}\right) Z=0 \\
{ }^{t}\left(\widetilde{N} \cdot V_{0}-Z_{N}\right) Z=0 .
\end{array}\right.
$$

Autrement dit,

$$
\left\{\begin{array}{l}
{ }^{t}\left(A_{T} V_{1}-Z_{T, 1}\right) Z=0 \\
{ }^{t}\left(\left(I+A_{T}^{\prime}\right) V_{2}-Z_{T, 2}\right) Z=0 \\
{ }^{t}\left(V_{2}-Z_{N, 1}\right) Z=0 .
\end{array}\right.
$$

Ce qui implique que la projection de $\left(I+A_{T}^{\prime}\right) Z_{N, 1}-Z_{T, 2}$ sur $F$ est nulle. Donc si pour tout sous espace $F$ de $R^{\prime}$ stable par $A_{T}^{\prime}$, la projection de $\left(I+A_{T}^{\prime}\right) Z_{N, 1}-Z_{T, 2}$ sur $F$ est non nulle, alors $\mathcal{H}$ est indécomposable. Supposons maintenant qu'il existe un sous-espace $F_{0} \neq 0$ de $R^{\prime}$ stable par $A_{T}^{\prime}$, telle que la projection de $\left(I+A_{T}^{\prime}\right) Z_{N, 1}-Z_{T, 2}$ sur $F_{0}$ est nulle. On remarque que la restriction $A_{T, F_{0}}$ de $A_{T}^{\prime}$ à $F_{0}$ est nulle (si non $\mathcal{H}$ laisse invariant $\left\{\left(-{ }^{t} V_{0} Z, Z, 0\right) / Z \in F_{1}\right\}$ avec $V_{0}=\left(\left(A_{T, F_{1}}\right)^{-1} Z_{T, 1, F_{1}}, Z_{N, 1, F_{1}}\right)$ $\left(Z_{T, 1, F_{1}}\right.$ est la projection de $Z_{T, 1}$ sur $F_{1}$ etc). Par suite $Z_{N, 1, F_{0}}=Z_{T, 2, F_{0}}$. On obtient donc 
Proposition 3.18. Si $\mu=0$, alors $A_{N}^{\prime}=0, \phi_{2}(\mathcal{D})=0, Z_{N, 2}=0$ et

Ou bien pour tout sous-espace $F \neq 0$ de $R^{\prime}$ stable par $A_{T}^{\prime}$, la projection de $\left(I+A_{T}^{\prime}\right) Z_{N, 1}^{\prime}-Z_{T, 2}^{\prime}$ sur $F$ est non nulle.

Ou bien il existe un sous-espace $F_{0} \neq 0$ de $R^{\prime}$ stable par $A_{T}^{\prime}$ telle que la projection de $\left(I+A_{T}^{\prime}\right) Z_{N, 1}^{\prime}-Z_{T, 2}^{\prime}$ sur $F_{0}$ est nulle. Et on a $A_{T, F_{0}}=0$. En plus $\mathcal{H}$ est indécomposable, si et seulement si, $F_{0}=\operatorname{vect}\left\{Z_{T, 1, F_{0}}^{\prime}\right\}$.

Des Propositions $2.1, \ldots, 3.18$ on obtient

Théorème 3.19. Pour $r=2$, soit $\mathcal{H}$ l'algèbre de Lie d'un sousgroupe $H$ de $S O_{0}(\langle\rangle)$ indécomposable, qui laisse invariant $\mathbb{R} e_{1}+\mathbb{R} e_{2}$ et qui contient l'idéal $\mathcal{B}$. Alors $H$ est dans $\mathbf{G}$ et

1) Ou bien $\mathcal{H}$ contient $\mathcal{P} \oplus \mathcal{B}$ avec $\mathcal{P}$ un sous-espace de $\mathcal{N}$ vérifiant $\mathcal{P}_{1}+\mathcal{P}_{2}=\mathbb{R}^{n}$ où $\mathcal{P}_{i}$ est la $i$-ème projection de $\mathcal{P}$ sur $\mathbb{R}^{n}$ pour $i=1,2$.

Ou bien on a l'un des cas suivants:

2) Il existe une sous-algèbre $\mathcal{U}^{\prime}$ de $g l(n, \mathbb{R})$, une décomposition non triviale $n=p+q$ telle que $\mathcal{H}$ contient un sous-espace $\mathcal{P}$ de $\mathcal{N}$ avec $\mathcal{P}_{1}+\mathcal{P}_{2}=\mathbb{R}^{p}$ et il existe une sous algèbre commutative $\mathcal{C}$ de so $(p, \mathbb{R})$, une sous-algèbre semi-simple $\mathcal{D}$ de so $(p, \mathbb{R})$ qui commute avec $\mathcal{C}$ et six applications linéaires $\varphi_{1}: \mathcal{U}^{\prime} \rightarrow \operatorname{so}(p, \mathbb{R}), \varphi_{2}: \mathcal{U}^{\prime} \rightarrow$ so $(q, \mathbb{R}), \psi: \mathcal{C} \oplus \mathcal{D} \rightarrow \mathbb{R}^{2 p}, \xi: \mathcal{U}^{\prime} \rightarrow \mathbb{R}^{2 p}, \phi: \mathcal{C} \rightarrow \mathbb{R}^{2 q}$, et $\eta: \mathcal{U}^{\prime} \rightarrow \mathbb{R}^{2 q}$ telles qu'a une conjuguaison près dans $\mathcal{G}$, on a l'un des sous cas suivants:

2.1) $\mathcal{H}$ est formée par les matrices "bloc"

$$
\left(\begin{array}{cccc}
U & { }^{t}(Y+\psi(A+B)+\xi(U)) & { }^{t}(\phi(A)+\eta(U)) & \delta J \\
0 & A+B+\varphi_{1}(U) & 0 & Y+\psi(A+B)+\xi(U)) \\
0 & 0 & \varphi_{2}(U) & \phi(A)+\eta(U) \\
0 & 0 & 0 & -{ }^{t} U
\end{array}\right)
$$

$A \in \mathcal{C}, B \in \mathcal{D}, Y \in \mathcal{P}$ et $\delta \in \mathbb{R}$. Avec $\phi_{1}(\mathcal{C})+\phi_{2}(\mathcal{C})=\mathbb{R}^{q}$ où $\phi_{1}(\mathcal{C})$ et $\phi_{2}(\mathcal{C})$ sont les composantes de $\phi(\mathcal{C})$ et tout élément non nul du centre de $\mathcal{U}^{\prime}$ admet au moins une valeur propre imaginaire pure ou nulle. 
2.2) Il existe une autre décomposition $q=q_{1}+q_{2}$, où $q_{2} \neq 0, \phi: \mathcal{C} \rightarrow$ $\mathbb{R}^{2 q_{1}}$ et $\mathcal{H}$ est formée par les matrices "bloc"

$$
\begin{gathered}
\left(\begin{array}{ccccc}
\alpha U_{0} & { }^{t}\left(Y+\psi(A+B)+\alpha Y_{0}\right) & { }^{t}\left(\phi(A)+\alpha Z_{0}\right) & \alpha^{t} Z_{0}^{\prime} & \delta J \\
0 & A+B+\alpha A_{0} & 0 & 0 & Y+\psi(A+B)+\alpha Y_{0} \\
0 & 0 & \alpha B_{0} & 0 & \phi(A)+\alpha Z_{0} \\
0 & 0 & 0 & \alpha C_{0} & \alpha Z_{0}^{\prime} \\
0 & 0 & 0 & 0 & -\alpha{ }^{t} U_{0}
\end{array}\right) \\
\multicolumn{4}{c}{\text { avec } \phi_{1}(\mathcal{C})+\phi_{2}(\mathcal{C})=\mathbb{R}^{q_{1}} \text { et }}
\end{gathered}
$$

2.2.1) $O u$ bien $U_{0}=J=\left(\begin{array}{cc}0 & 1 \\ -1 & 0\end{array}\right), C_{0}=\left(\begin{array}{ccc}\varepsilon_{1} J & & 0 \\ & \ddots & \\ 0 & & \varepsilon_{l} J\end{array}\right), \varepsilon_{k}= \pm 1$ pour $k=1, \ldots, l=\frac{q_{2}}{2}$ et $C_{0} Z_{1, F}^{\prime} \neq Z_{2, F}^{\prime}$ pour tout sousespace $F$ de $\mathbb{R}^{q_{1}}$ invariant par $C_{0}$. Avec $Z_{0}^{\prime}=\left(Z_{1}^{\prime}, Z_{2}^{\prime}\right), Z_{j, F}^{\prime}$ la projection de $Z_{j}^{\prime}$ sur $F$, pour $j=1,2$.

2.2.2) $O u$ bien $U_{0}=N, C_{0}=0, q_{2}=1$ et $Z_{0}^{\prime}=(a, b)$ avec $b \neq 0$ $\left(N=\left(\begin{array}{ll}0 & 1 \\ 0 & 0\end{array}\right)\right)$.

2.2.3) Ou bien $U_{0}=S, C_{0}=0, \phi_{1}(\mathcal{C})=0$ et $Z_{0}^{\prime}=(a, b)$ avec $b \neq 0$ $\left(S=\left(\begin{array}{ll}1 & 0 \\ 0 & 0\end{array}\right)\right)$.

2.3) $q=1$ ou 2 et $\mathcal{H}$ est formée par les matrices "bloc"

$$
\left(\begin{array}{cccc}
\alpha U_{0}+\beta U_{0}^{\prime} & { }^{t}\left(Y+\psi(A+B)+\alpha Y_{0}+\beta Y_{0}^{\prime}\right) & { }^{t}\left(\alpha Z_{0}+\beta Z_{0}^{\prime}\right) & \delta J \\
0 & A+B+\alpha A_{0}+\beta B_{0} & 0 & Y+\psi(A+B)+\alpha Y_{0}+\beta Y_{0}^{\prime} \\
0 & 0 & 0 & \alpha Z_{0}+\beta Z_{0}^{\prime} \\
0 & 0 & 0 & -{ }^{t}\left(\alpha U_{0}+\beta U_{0}^{\prime}\right)
\end{array}\right)
$$

avec $Z_{0}, Z_{0}^{\prime} \in \mathbb{R}^{2 q}$ et

2.3.1) Ou bien $U_{0}=N, U_{0}^{\prime}=S$ et $0 \times \mathbb{R}^{q}$ est engendré par $Z_{0,2}$ et $Z_{0,2}^{\prime}$.

2.3.2) $O u$ bien $U_{0}=N, U_{0}^{\prime}=T_{\mu}(\mu \neq 0,1,2)$ et si $Z_{0}=(a, b)$, $Z_{0}^{\prime}=(c, a)$ avec $a \neq 0\left(T_{\mu}=\left(\begin{array}{cc}\mu & 0 \\ 0 & 1\end{array}\right)\right)$. 
2.4) $\mathcal{H}$ est formée par les matrices "bloc"

$$
\left(\begin{array}{ccccc}
\alpha N+\beta T_{0} & { }^{t}(\psi(A+B)+ & { }^{t}(\phi(A)+ & & \\
& \left.Y+\alpha Y_{N}+\beta Y_{T}\right) & \left.\alpha Z_{N}+\beta Z_{T}\right) & \left(\alpha Z_{N}^{\prime}+\beta Z_{T}^{\prime}\right) & \delta J \\
0 & A+B & 0 & 0 & \psi(A+B)+ \\
0 & +\alpha A_{N}+\beta A_{T} & 0 & 0 & \phi(A)+\alpha Z_{N}+\beta Z_{T} \\
0 & 0 & \alpha B_{N}+\beta B_{T} & 0 & \alpha Y_{T} \\
0 & 0 & 0 & 0 & -{ }^{t}\left(\alpha N+\beta Z_{0}^{\prime}+\beta Z_{T}^{\prime}\right.
\end{array}\right)
$$

$\phi_{2}(\mathcal{C})=0, \phi_{2}(\mathcal{C})=\mathbb{R}^{q}, Z_{N, 2}^{\prime}=0$ et

2.4.1) Ou bien pour tout sous-espace $F \neq 0$ de $\mathbb{R} e_{s+2}+\cdots+\mathbb{R} e_{n+2}$ stable par $C_{T}$ la projection de $\left(I+C_{T}\right) Z_{N, 1}^{\prime}-Z_{T, 2}^{\prime}$ sur $F$ est non nulle $\left(s=n-q_{2}+1\right)$.

2.4.2) Ou bien $Z_{N, 1}^{\prime}$ et $Z_{T, 2}^{\prime}$ ont la même $(n+2)$-ième coordonnée et la $(n+2)$-ième coordonnée de $Z_{T, 1}^{\prime}$ est non nulle. En plus

$$
C_{T}=\left(\begin{array}{cc}
C_{T}^{\prime} & 0 \\
0 & 0
\end{array}\right)
$$

avec $C_{T}^{\prime} \in \operatorname{gl}\left(q_{2}-1, \mathbb{R}\right)$ et la projection sur $F^{\prime}$ de $(I+$ $\left.C_{T}\right) Z_{N, 1}^{\prime}-Z_{T, 2}^{\prime}$ est non nulle pour tout sous espace $F^{\prime} \neq 0$ de $\mathbb{R} e_{s+2}+\cdots+\mathbb{R} e_{n+1}$ stable par $C_{T}^{\prime}$.

Remarque. La réalisation géométrique d'un groupe comme groupe d'holonomie d'une variété affine est l'étape la plus difficile du problème d'holonomie. Pour le cas des variétés irréductibles, il a fallu attendre plus que trente ans pour réaliser la liste de M. Berger (voir [5]). Malheuresement, la plus part des techniques utilisées ne sont valables que pour le cas irréductible. Ce qui nécessite des techniques différentes pour la réalisation de la liste du Théorème 3.19. Mais au moins localement, en utilisant le Théorème d'Ambrose-Singer et le Théorème 5.27 qui donne un système de coordonnées adapté à notre holonomie, on arrive à réaliser certains groupes de la liste du Théorème 3.19 (voir le paragraphe 6 ).

\section{Cas des espaces pseudo-riemanniens symétriques}

Dans ce paragraphe, on fait quelques rappels sur les espaces pseudoriemanniens symétriques. 
Soit $(M, g)$ un espace pseudo-riemannien symétrique connexe indécomposable et non irréductible. Soit $\mathcal{L}$ l'algèbre du groupe des transvections de $(M, g), \sigma$ la différentielle de la conjuguaison par la symétrie $s_{\theta}$ $(\theta$ un point de base de $M$ ) et $\eta$ l'extension à $\mathcal{L}$ de la 2 -forme symétrique sur $T_{\theta} M$ induite par $g_{\theta}$. Si on pose $\mathcal{L}^{\prime}=\{X \in \mathcal{L} / \sigma X=X\}$ et $\mathcal{L}^{\prime \prime}=$ $\{X \in \mathcal{L} / \sigma X=-X\}$, alors $\mathcal{L}^{\prime \prime}$ est isomorphe à $T_{\theta} M$ et on a: $\mathcal{L}^{\prime}=$ $\left[\mathcal{L}^{\prime \prime}, \mathcal{L}^{\prime \prime}\right]$. L'algèbre de Lie $\mathcal{H}$ de l'holonomie de $(M, g)$ est la représentation fidèle de $a d \mathcal{L}^{\prime}$ dans $\mathcal{L}^{\prime \prime}$. Le centre $\mathcal{Z}$ de $\mathcal{L}$ est donné par (voir [13, ch. 1, Lemme 1]):

$$
\mathcal{Z}=\left\{X \in \mathcal{L}^{\prime} \text { tel que } \mathcal{H} \cdot X=0\right\}=\left[\mathcal{L}^{\prime}, \mathcal{L}^{\prime \prime}\right] \cap\left[\mathcal{L}^{\prime}, \mathcal{L}^{\prime \prime}\right]^{\perp}
$$

$(M, g)$ est supposée indécomposable donc $\mathcal{Z}$ est totalement isotrope ou nul. D'autre part, on a:

Proposition $4.20([\mathbf{1 3}])$. L est résoluble si et seulement si la représentation d'isotropie de $\mathcal{H}$ est nilpotente. Et $\mathcal{L}$ est semi-simple si et seulement si la représentation d'isotropie de $\mathcal{H}$ est complètement réductible.

M. Berger a donné dans [6] la liste complète des espaces pseudoriemanniens symétriques lorsque la représentation d'holonomie est complètement réductible. Le cas où $\mathcal{L}$ n'est ni semi-simple ni résoluble a été étudié par M. Cahen et M. Parker [13] (en signature quelconque). En particulier, ils ont donné une classification complète en dimension $\leq 7$. Pour le cas de la signature $(2,2+n)$, M. Parker a donné une classification complète dans $[\mathbf{2 0}]$. Enfin, le cas où $L$ est résoluble a également été étudié par Cahen et Parker dans [12], avec une classification complète du cas de signature $(2,2+n)$. Le cas de signature $(2,2+n)$ est donc contenu dans ces classifications.

Dans ce qui suit, lorsque $L$ n'est pas semi-simple, on précise simplement, par rapport aux notations du Théorème 3.19 les possibilités du groupe d'holonomie restreint de tout espace pseudo-riemannien symétrique de signature $(2,2+n)$ indécomposable non irréductible. Et on donnera des exemples de ces espaces. Cependant, pour faciliter la lecture de cet article, on rappellera quelques détails de ces classifications dans notre cas particulier.

On suppose aussi que le groupe de transvections de $(M, g)$ est non semi-simple et on distinguera deux cas:

\section{Cas où $\mathcal{L}$ est résoluble.}

$\mathcal{H}$ est donc nilpotente et $\mathcal{Z} \neq 0$. 
Proposition 4.21. À un isomorphisme près, l'algèbre de Lie $\mathcal{H}$ ne peut être que l'un des cas suivants:

a) Si $\mathcal{Z}$ est de dimension $1, \mathcal{P} \subset \mathcal{H} \subset \mathcal{P} \oplus \mathcal{B}$ avec $\mathcal{P}_{1}+\mathcal{P}_{2}=\mathbb{R}^{n}$. Si $\mathcal{Z}$ est de dimension 2 ,

b) Ou bien $\mathcal{U}^{\prime} \oplus \mathcal{P} \subset \mathcal{H} \subset \mathcal{U}^{\prime} \oplus \mathcal{P} \oplus \mathcal{B}$ où $\mathcal{U}^{\prime} \in\{\mathbb{R} S, \mathbb{R} N\}$ avec $\mathcal{P}_{1}+\mathcal{P}_{2}=\mathbb{R}^{n}$.

c) Ou bien

$$
\mathcal{H}=\left\{\left(\begin{array}{cccc}
\alpha U_{0} & { }^{t} Y & \alpha^{t} Z_{0} & \delta J \\
0 & 0 & 0 & Y \\
0 & 0 & 0 & \alpha Z_{0} \\
0 & 0 & 0 & -\alpha^{t} U_{0}
\end{array}\right), \alpha, \delta \in \mathbb{R}, Y \in P^{\prime}\right\}
$$

avec $U_{0}=S$ ou $N, Z_{0}=(a, b) \in \mathbb{R}^{2}$ où $b \neq 0$ et $P^{\prime}$ un sous-espace de $\mathbb{R}^{2 n-2}$.

Preuve: D'après (13), on peut écrire $\left[\mathcal{L}^{\prime}, \mathcal{L}^{\prime \prime}\right]=\mathcal{Z} \oplus W$, avec $W$ non dégénéré vérifiant $\left[\mathcal{L}^{\prime}, W\right] \subset \mathcal{Z},[W, W]=0$ et $\mathcal{L}^{\prime \prime}=\mathcal{Z} \oplus W \oplus \mathcal{Z}^{*}$ où $\mathcal{Z}^{*}$ est le dual de $\mathcal{Z}$ relativement à $\eta$. Si $\mathcal{Z}$ est maximal, à un isomorphisme près, on peut supposer $\mathcal{Z}=\mathbb{R} e_{1} \oplus \mathbb{R} e_{2}, \mathcal{Z}^{*}=\mathbb{R} e_{n+3} \oplus \mathbb{R} e_{n+4}$ et $W=\mathbb{R} e_{3} \oplus$ $\cdots \oplus \mathbb{R} e_{n+2}$. Donc, $\mathcal{H} \subset \mathcal{G}$ avec $\mathcal{U}^{\prime}=0$. D'où d'après le Théoreme 3.17, $\mathcal{H}$ ne peut être que de type 1 ou 2.1. Si $\mathcal{H}$ est de type 1 , elle contient $\mathcal{P}$ avec $\mathcal{P}_{1}+\mathcal{P}_{2}=\mathbb{R}^{n}$ et puisque $\mathcal{H}$ est nilpotente, il existe un entier $s \neq 0$ tel que pour $(0, A, 0,0) \in \mathcal{K}^{\prime}$ et $(0,0, X, 0) \in \mathcal{P}$, d'après $(2)$, on a:

$$
a d^{s}(0, A, 0,0) \cdot(0,0, X, 0)=\left(0,0, \widetilde{A}^{s} X, 0\right)=0 .
$$

Ce qui implique que $A^{s} \mathcal{P}_{1}=A^{s} \mathcal{P}_{2}=0$. Or $\mathcal{K}^{\prime}$ est une sous algèbre nilpotente de $s o(n)$, donc elle est commutative. D'après ce qui precède, on en déduit $\mathcal{K}^{\prime}=0$.

Si $\mathcal{H}$ est de type 2.1 , elle contient $\mathcal{P}$ avec $\mathcal{P}_{1}+\mathcal{P}_{2}=\mathbb{R}^{p}$ et il existe un entier $s \neq 0$ tels que:

$$
a d^{s}(0, A, \omega(A), 0) \cdot(0,0, X, 0)=\left(0,0, \widetilde{A}^{s} X,\left\langle\omega(A), \widetilde{A}^{s-1} X\right\rangle_{J}\right)=0
$$

et pour $(0, A, \omega(A), 0) \in \mathcal{H}$ avec $A \in \mathcal{K}^{\prime} \subset$ so $(p)$ et $(0,0, X, 0) \in \mathcal{P}$. On en déduit donc $\mathcal{K}^{\prime}=0$. Maintenant, si $\mathcal{Z}$ est de dimension 1 , à un isomorphisme près, ou bien $\mathcal{Z}=\mathbb{R} e_{1}, \mathcal{Z}^{*}=\mathbb{R} e_{n+3}$ et $W=\mathbb{R} e_{2} \oplus \mathbb{R} e_{3} \oplus$ $\cdots \oplus \mathbb{R} e_{n+2} \oplus \mathbb{R} e_{n+4}$, donc $\mathcal{U}^{\prime}=\mathbb{R} N$. Ou bien $\mathcal{Z}=\mathbb{R} e_{2}, \mathcal{Z}^{*}=\mathbb{R} e_{n+4}$ et $W=\mathbb{R} e_{1} \oplus \mathbb{R} e_{3} \oplus \cdots \oplus \mathbb{R} e_{n+2} \oplus \mathbb{R} e_{n+3}$, donc $\mathcal{U}^{\prime}=\mathbb{R} S$. Par conséquent $\mathcal{H}$ est de type 1 ou 2.1 ou 2.2 .2 ou 2.2.3. De la même façon que ci-dessus, on montre que $\mathcal{K}^{\prime}=0$. 
Cas où $\mathcal{L}$ n'est ni semi-simple ni résoluble.

Soit $\mathcal{L}=\mathcal{S} \oplus \mathcal{R}$ une décomposition de Levi de $\mathcal{L}$ invariante par $\sigma$, avec $\mathcal{S}$ sa partie semi-simple et $\mathcal{R}$ son radical. On peut donc écrire

$$
\mathcal{L}=\mathcal{L}_{R}^{\prime} \oplus \mathcal{L}_{S}^{\prime} \oplus \mathcal{L}_{R}^{\prime \prime} \oplus \mathcal{L}_{S}^{\prime \prime}
$$

avec $\mathcal{L}_{R}^{\prime}=\mathcal{L}^{\prime} \cap \mathcal{R}, \mathcal{L}_{S}^{\prime}=\mathcal{L}^{\prime} \cap \mathcal{S}, \mathcal{L}_{R}^{\prime \prime}=\mathcal{L}^{\prime \prime} \cap \mathcal{R}$, et $\mathcal{L}_{S}^{\prime \prime}=\mathcal{L}^{\prime \prime} \cap \mathcal{S}$.

Proposition 4.22 (voir [13, Théorème 1.1, ch. 2]). $\mathcal{L}^{\prime \prime}=\mathcal{L}_{R}^{\prime \prime} \oplus \mathcal{L}_{S}^{\prime \prime}=$ $X \oplus W \oplus \mathcal{L}_{S}^{\prime \prime}$ avec $X=\mathcal{L}_{R}^{\prime \prime} \cap\left(\mathcal{L}_{R}^{\prime \prime}\right)^{\perp}$ le dual de $\mathcal{L}_{S}^{\prime \prime}$ relativement à $\eta$ et $W=\mathcal{L}^{\prime \prime} \cap\left(X \oplus \mathcal{L}_{S}^{\prime \prime}\right)^{\perp}$ non dégénéré.

Proposition 4.23 (voir [13, Corollaire 1.7, ch. 2]). Tout espace symétrique $(M, g)$ de signature $(2,2+n)$ admet "une multiplication minimale", c'est à dire $\left[\left[\mathcal{L}_{R}^{\prime \prime}, \mathcal{L}_{R}^{\prime \prime}\right], \mathcal{L}_{R}^{\prime \prime}\right]=0$, et on a:

a) Le centre $\mathcal{Z}$ de $\mathcal{L}$ est nul.

b) $\mathcal{S}$ est de dimension 3 .

c) La restriction de $\eta$ à $W$ est définie négative.

d) $\left[\mathcal{L}_{R}^{\prime}, W\right] \subset X$.

On en déduit donc que $X$ et $\mathcal{L}_{S}^{\prime \prime}$ sont de dimension 2 et que $\mathcal{L}_{S}^{\prime}$ est de dimension 1. Par conséquent, à un isomorhpisme près, $X=\mathbb{R} e_{1} \oplus \mathbb{R} e_{2}$,

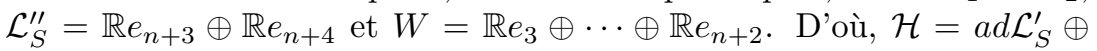
$\operatorname{ad\mathcal {L}_{R}^{\prime }} \subset \mathcal{G}$ et on a $a d \mathcal{L}_{R}^{\prime} \subset \mathcal{N} \oplus \mathcal{B}$. Et puisque $\left[\mathcal{L}_{S}^{\prime}, \mathcal{L}_{S}^{\prime \prime}\right] \subset \mathcal{L}_{S}^{\prime \prime}$, et $\mathcal{Z}=0$, alors $a d \mathcal{L}_{S}^{\prime}$ est une droite engendrée par $\left(U_{0}, 0,0,0\right)$ avec $U_{0}$ inversible. On obtient donc

Proposition 4.24. L'algèbre de Lie $\mathcal{H}$ de l'holonomie d'un espace symétrique connexe de type $(2, n+2)$ à groupe de transvections $\mathcal{L}$ non semi-simple et non résoluble ne peut être à un isomorphisme près, que incluse dans $\mathcal{U}^{\prime} \oplus \mathcal{P} \oplus \mathcal{B}$ où $\mathcal{U}^{\prime}$ est engendrée par une matrice inversible $U_{0}$ et $\mathcal{P}$ vérifiant $\mathcal{P}_{1}+\mathcal{P}_{2}=\mathbb{R}^{n}$.

\subsection{Exemples.}

Soit $(M, g)$ un espace symétrique connexe de signature $(2, n+2)$, indécomposable et non irréductible. On note $R$ son tenseur de courbure. On sait que pour $\theta$ un point de $M, R$ est défini en $\theta$ par $R(X, Y)=$ $-a d[X, Y]$ pour $X, Y \in T_{\theta} M$.

Nous donnerons dans la suite deux exemples d'espaces symétriques indécomposables et non irréductibles qui sont totalement caractérisés par leurs groupes d'holonomie restreints. 
Exemple 1. Avec les mêmes notations ci-dessus, nous supposons que le centre $\mathcal{Z}$ de $\mathcal{L}$ est maximal. En vertu de la Proposition 4.20 -a, $\mathcal{H} \subset$ $\mathcal{P} \oplus \mathcal{B}$ avec $\mathcal{P}_{1}+\mathcal{P}_{2}=\mathbb{R}^{n}$. Nous supposons en plus que $\mathcal{P}_{2}=0$ et $\mathcal{H}=$ $\mathcal{P} \oplus \mathcal{B}$. Donc les seuls crochets non nuls dans $\mathcal{L}^{\prime}$ sont $Y=\left[e_{n+3}, e_{n+4}\right]$, $U_{i}=\left[e_{n+3}, e_{i}\right](i=3, \ldots, n+2)$. Ce qui implique $\mathcal{L}^{\prime}$ est une sous-algèbre commutative, engendrée par $\left\{Y, U_{i}\right\}$. Si on pose $a d Y=\left(0,0,\left(Y_{1}, 0\right), \delta\right)$, et $a d U_{i}=\left(0,0,\left(C_{i}, 0\right), \delta_{i}\right)$ avec $Y_{1}=\left(a_{1}, \ldots, a_{n}\right), C_{i}=\left(c_{i 1}, \ldots, c_{i n}\right)$, il est immédiat que la matrice $C=\left(c_{i j}\right)$ est symétrique et $\delta_{i}=a_{i}$. Puisque $\mathcal{H}$ est indécomposable et contient $\mathcal{B}$, alors $\left\{Y_{1}, C_{i}\right\}$ engendre $\mathbb{R}^{n}$ et $\left(Y_{1}, \delta\right) \neq 0$. Or $\mathcal{H}$ est de dimension $n+1$, donc $\left\{Y, U_{i}\right\}$ est une base de $\mathcal{L}^{\prime}$. Par conséquent, on peut identifier $\mathcal{L}^{\prime}$ à $\mathbb{R}^{n} \times \mathbb{R}$ et $\mathcal{L}$ à $\mathcal{T}=\mathbb{R}^{2 n+5}=\mathbb{R} \times \mathbb{R} \times \mathbb{R}^{n} \times \mathbb{R} \times \mathbb{R} \times \mathbb{R}^{n} \times \mathbb{R}$ munie du crochet

$$
\left[(x, y, X, z, t, Z, u),\left(x^{\prime}, y^{\prime}, X^{\prime}, z^{\prime}, t^{\prime}, Z^{\prime}, u^{\prime}\right)\right]=\left(x^{\prime \prime}, y^{\prime \prime}, X^{\prime \prime}, 0,0, Z^{\prime \prime}, u^{\prime \prime}\right),
$$

où

$$
\begin{aligned}
x^{\prime \prime}= & \left\langle C X^{\prime}, Z\right\rangle-\left\langle C X, Z^{\prime}\right\rangle \\
& +\left\langle u X^{\prime}-u^{\prime} X+t^{\prime} Z-t Z^{\prime}, Y_{1}\right\rangle+\delta\left(u t^{\prime}-u^{\prime} t\right), \\
y^{\prime \prime}= & \left\langle z Z^{\prime}-z^{\prime} Z, Y_{1}\right\rangle+\delta\left(z u^{\prime}-z^{\prime} u\right), \\
X^{\prime \prime}= & z^{\prime}\left(C Z+u Y_{1}\right)-z\left(C Z^{\prime}+u^{\prime} Y_{1}\right), \\
Z^{\prime \prime}= & \left(t^{\prime}-z^{\prime}\right) X-(t-z) X^{\prime} \text { et } u^{\prime \prime}=z t^{\prime}-z^{\prime} t,
\end{aligned}
$$

$(\langle$,$\rangle est le produit scalaire ordinaire).$

Inversement, on se donne une matrice symétrique réelle $C$ d'ordre $n$, $Y_{1} \in \mathbb{R}^{n}$ et $\delta \in \mathbb{R}$ telles que $\left\{Y_{1}, C_{i}\right\}$ engendre $\mathbb{R}^{n}$ et $\left(Y_{1}, \delta\right) \neq 0$. Si $\mathcal{T}$ est définie par (14), $\mathcal{T}^{\prime}=\{(0,0,0,0,0, Z, u)\}$ est une sous-algèbre abéliènne de $\mathcal{T}$ et $\mathcal{T}^{\prime \prime}=\{(x, y, X, z, t, 0,0)\}$ est un supplémentaire de $\mathcal{T}^{\prime}$ dans $\mathcal{T}$ $\operatorname{ad} \mathcal{T}^{\prime}$-invariant. La 2 -forme symétrique sur $\mathcal{T}^{\prime \prime}$

$\varphi\left((x, y, X, z, t, 0,0),\left(x^{\prime}, y^{\prime}, X^{\prime}, z^{\prime}, t^{\prime}, 0,0\right)\right)=x z^{\prime}+x^{\prime} z+y t^{\prime}+y^{\prime} t-\left\langle X, X^{\prime}\right\rangle$

est non dégénérée, de signature $(2,2+n)$ et ad $\mathcal{T}^{\prime}$-invariant. On note $T$ le sous-groupe résoluble simplement connexe engendré par $\mathcal{T}$ et $T^{\prime}$ le sous-groupe fermé correspondant à $\mathcal{T}^{\prime}$. Donc $\varphi$ induit une métrique $h$ de signature $(2,2+n)$ sur $M^{\prime}=T / T^{\prime}$ et on a:

Proposition 4.25. La variété $\left(M^{\prime}, h\right)$ est un espace symétrique de signature $(2,2+n)$, simplement connexe, indécomposable et non irréductible. Et tout espace localement symétrique de signature $(2,2+n)$, indécomposable et non irréductible dont l'algèbre d'holonomie restreint est $\mathcal{P} \oplus \mathcal{B}$ avec $\mathcal{P}_{1}=\mathbb{R}^{n}$ et $\mathcal{P}_{2}=0$ est isométrique à l'un de ces espaces. 
Exemple 2. On suppose que $\mathcal{H}=\{(\alpha N, 0,(X, 0), \delta) / \alpha, \delta \in \mathbb{R}$, $\left.X \in \mathbb{R}^{n}\right\}$. Donc les seuls crochets non nuls dans $\mathcal{L}^{\prime}$ sont $Y=\left[e_{n+3}, e_{n+4}\right]$, $Y^{\prime}=\left[e_{2}, e_{n+3}\right]$ et $U_{i}=\left[e_{i}, e_{n+3}\right](i=3, \ldots, n+2)$. On pose $a d Y=$ $\left(\alpha N, 0,\left(Y_{1}, 0\right), \delta\right), a d Y^{\prime}=\left(\alpha^{\prime} N, 0,\left(Y_{2}, 0\right), \delta^{\prime}\right)$ et $a d U_{i}=\left(\alpha_{i}, 0,\left(C_{i}, 0\right), \delta_{i}\right)$ avec $Y_{1}=\left(a_{1}, \ldots, a_{n}\right), Y_{2}=\left(b_{1}, \ldots, b_{n}\right)$, et $C_{i}=\left(c_{i 1}, \ldots, c_{i n}\right)$, donc la matrice $C=\left(c_{i j}\right)$ est symétrique, $\delta_{i}=-a_{i}$ et $\alpha_{i}=b_{i}$. En plus $\left\{Y_{1}, Y_{2}, C_{i}\right\}$ engendre $\mathbb{R}^{n}$ et $\left(Y_{1}, \alpha\right) \neq 0$. Puisque $\mathcal{L}^{\prime}$ est commutative et de dimension $n+2$, on peut identifier $\mathcal{L}$ à $\mathcal{T}=\mathbb{R}^{2 n+5}=\mathbb{R} \times \mathbb{R} \times \mathbb{R}^{n} \times$ $\mathbb{R} \times \mathbb{R} \times \mathbb{R}^{n} \times \mathbb{R} \times \mathbb{R}$ munie du crochet

$$
\begin{aligned}
& {\left[(x, y, X, z, t, Z, u, v),\left(x^{\prime}, y^{\prime}, X^{\prime}, z^{\prime}, t^{\prime}, Z^{\prime}, u^{\prime}, v^{\prime}\right)\right]} \\
& =\left(x^{\prime \prime}, y^{\prime \prime}, X^{\prime \prime}, 0, t^{\prime \prime}, Z^{\prime \prime}, u^{\prime \prime}, v^{\prime \prime}\right),
\end{aligned}
$$

où

$$
\begin{aligned}
x^{\prime \prime}= & \left\langle C X^{\prime}, Z\right\rangle-\left\langle C X, Z^{\prime}\right\rangle \\
& +\left\langle u X^{\prime}-u^{\prime} X+t Z^{\prime}-t^{\prime} Z, Y_{1}\right\rangle+\left\langle v X^{\prime}-v^{\prime} X, Y_{2}\right\rangle \\
& +\alpha\left(u y^{\prime}+t v^{\prime}-u^{\prime} y-t^{\prime} v\right)+\alpha^{\prime}\left(v y^{\prime}-v^{\prime} y\right)+\delta\left(u t^{\prime}-u^{\prime} t\right), \\
y^{\prime \prime}= & \left\langle z^{\prime} Z-z Z^{\prime}, Y_{1}\right\rangle+\delta\left(z u^{\prime}-z^{\prime} u\right)+\alpha\left(v z^{\prime}-v^{\prime} z\right), \\
X^{\prime \prime}= & z^{\prime}\left(C Z+u Y_{1}+v Y_{2}\right)-z\left(C Z^{\prime}+u^{\prime} Y_{1}+v^{\prime} Y_{2}\right), \\
Z^{\prime \prime}= & z^{\prime} X-z X^{\prime}, u^{\prime \prime}=z t^{\prime}-z^{\prime} t, \text { et } v^{\prime \prime}=y z^{\prime}-y^{\prime} z .
\end{aligned}
$$

On pose maintenant $M^{\prime}=T / T^{\prime}$ avec $T$ le sous-groupe résoluble simplement connexe engendré par $\mathcal{T}, T^{\prime}$ le sous-groupe fermé correspondant à $\mathcal{T}^{\prime}=\{(0,0,0,0,0, Z, u, v)\}$ et $\varphi$ la 2 -forme symétrique définie sur $\mathcal{T}^{\prime \prime}=\{(x, y, X, z, t, 0,0)\}$ comme ci-dessus. $\varphi$ induit sur $M^{\prime}$ une métrique $h$ de signature $(2,2+n)$ et on a:

Proposition 4.26. La variété $\left(M^{\prime}, h\right)$ est un espace symétrique de signature $(2,2+n)$, simplement connexe, indécomposable, et non irréductible. Et tout espace localement symétrique de signature $(2,2+n)$, indécomposable et non irréductible tel que $\mathcal{H}=\{(\alpha N, 0,(X, 0), \delta) / \alpha, \delta \in \mathbb{R}$, $\left.X \in \mathbb{R}^{n}\right\}$ est isométrique à l'un de ces espaces.

\section{Construction d'un système de coordonnées}

Dans ce paragraphe, on se donne une métrique indécomposable non irréductible et on cherche à construire un système de coordonnées adapté à cette métrique. Plus précisement, on cherche à réduire localement la forme d'une métrique pseudo-riemannienne dont l'holonomie laisse 
invariant un sous-espace isotrope maximal. Ce qui nous faciletera à construire dans le dernier paragraphe des métriques dont l'holonomie vérifie les conclusions du Théorème 3.19 .

Pour cela, soit $(M, g)$ une variété pseudo-riemannienne de signature $(r, r+n)$ et de dimension $m=n+2 r$. Soit $\theta$ un point fixe de $M$ et $\left(e_{1}, \ldots, e_{r}, \varepsilon_{1}, \ldots, \varepsilon_{n}, e_{1}^{\prime}, \ldots, e_{r}^{\prime}\right)$ une base de $T_{\theta} M$ dans laquelle la métrique $g$ a pour forme au point $\theta$ :

$$
g(X, X)=\sum_{i=1}^{r} x_{i} z_{i}-\sum_{j=1}^{n} y_{j}^{2}, \text { pour } X=\sum_{i=1}^{n} x_{i} e_{i}+\sum_{i=1}^{n} y_{i} \varepsilon_{i}+\sum_{i=1}^{n} z_{i} e_{i}^{\prime} .
$$

On suppose que le groupe d'holonomie restreint $H$ de $(M, g)$ au point $\theta$ laisse invariant $\Re=\mathbb{R} e_{1}+\cdots+\mathbb{R} e_{r}$. Donc il laisse invariant aussi son orthogonal $\Re^{\perp}=\Re \oplus \mathbb{R} \varepsilon_{1}+\cdots+\mathbb{R} \varepsilon_{n}$. Par conséquent, on peut construire, par transport parallèle, deux distributions $T^{0}$ et $T$ telles que $T_{\theta}^{0}=\Re$ et $T_{\theta}=\Re^{\perp}$. En plus on a $D_{U} X \in T^{0}$ et $D_{U} Y \in T$ pour $X \in T^{0}, Y \in T$ et $U \in T M$. Les distributions $T^{0}$ et $T$ sont involutives, donc d'après Frobenuis il existe deux sous-variétés intégrales maximales $M_{0}$ et $M^{\prime}$ passant par $\theta$ associées respectivement à $T^{0}$ et $T$. On pose $M^{\prime \prime}$ l'espace quotient $M / T$ de $M$ sous la relation d'équivalence qui identifie deux points qui appartiennent à une même sous variété $M^{\prime}$. Si on remplace $M$ par un voisinage ouvert de $\theta, M^{\prime \prime}$ est une sous-variété de $M$ de dimension $r$. On note $\pi$ la projection $M \longrightarrow M^{\prime \prime}$. On pose $V$ le voisinage normale associé aux $e_{i}^{\prime}$ et on met $\left(z_{1}, \ldots, z_{r}\right)$ comme coordonnées sur $V$. On peut mettre ces mêmes coordonnées sur $M^{\prime \prime}$ en identifiant $M^{\prime \prime}$ à $V$. On transporte $T_{\theta}^{0}$ et $T_{\theta}$ le long de $V$ et on choisit en chaque point $z$ de $V$ un supplémentaire orthogonal $T_{z}^{\prime}$ de $T_{z}^{0}$ tel que la métrique restreinte à $T_{z}^{\prime}$ est définit négative.

Pour $z \in V$, on note $W_{z}$ le voisinage normale de $z$ associé au sousespace $T_{z}^{\prime}$. Et on note $P$ le sous-espace engendré par les $W_{z} . P$ contient $V$. On met des coordonnées sur $P$ comme suit:

Soit $y \in P, y$ appartient à un seul $W_{z}$. On ramène $y$ à $z$ par des coordonnées normales $\left(y_{1}, \ldots, y_{r}\right)$ dans $W_{z}$. Puis on ramène $z$ à l'origine par les coordonnées $\left(z_{1}, \ldots, z_{r}\right)$. Maintenant on choisit le long de $P, r$ champs de vecteurs $E_{i} \in T^{0}$ tels que:

$$
g\left(E_{i}, \frac{\partial}{\partial z_{j}}\right)=\delta_{i j} \text { pour } i, j=1, \ldots, r .
$$

Or si on note $R$ le tenseur de courbure de $g$, on a pour $U, V \in T M$ et $X, Y \in T^{0}, g(R(X, Y) U, V)=g(R(U, V) X, Y)=0$. Par conséquent $R$ est nul sur $M_{0}$. Donc on peut choisir des coordonnées $\left(x_{1}, \ldots, x_{r}\right)$ dans 
$M_{0}$ telles que $E_{i}=\frac{\partial}{\partial x_{i}}$ et $g\left(\frac{\partial}{\partial x_{i}}, \frac{\partial}{\partial x j}\right)=0$. Enfin on peut mettre des coordonnées sur un voisinage de $\theta$, comme suit:

Soit $x$ un point de $M$ voisin de $\theta$, il passe par $x$ une seule sous-variété $M_{0}$ qui rencontre l'espace $P$ en un seul point $y$. On ramène $x$ à $y$ par les coordonnées $\left(x_{1}, \ldots, x_{r}\right)$, puis on prend pour les autres coordonnées de $x$ celles de $y$.

Maintenant on regarde les propriétés des champs de vecteurs $\frac{\partial}{\partial x_{i}}, \frac{\partial}{\partial y_{j}}$ et $\frac{\partial}{\partial z_{k}}$. D'abord par construction on a partout:

$$
D_{\frac{\partial}{\partial x_{i}}} \frac{\partial}{\partial x_{j}}=0 \text { et } g\left(\frac{\partial}{\partial x_{i}}, \frac{\partial}{\partial x_{j}}\right)=0 \text { pour } i, j=1, \ldots, r \text {. }
$$

Le long de $P$, on a $g\left(\frac{\partial}{\partial x_{i}}, \frac{\partial}{\partial z_{j}}\right)=\delta_{i j}$, en plus on a:

$$
\frac{\partial}{\partial x_{k}} g\left(\frac{\partial}{\partial x_{i}}, \frac{\partial}{\partial z_{j}}\right)=g\left(D_{\frac{\partial}{\partial x_{k}}} \frac{\partial}{\partial x_{i}}, \frac{\partial}{\partial z_{j}}\right)+g\left(\frac{\partial}{\partial x_{i}}, D_{\frac{\partial}{\partial x_{k}}} \frac{\partial}{\partial z_{j}}\right)=0
$$

car $D_{\frac{\partial}{\partial x_{j}}} \frac{\partial}{\partial x_{i}}=0$ et $D_{\frac{\partial}{\partial x_{k}}} \frac{\partial}{\partial z_{j}}=D_{\frac{\partial}{\partial z_{j}}} \frac{\partial}{\partial x_{k}} \in T^{0}$. Ce qui implique donc que $g\left(\frac{\partial}{\partial x_{i}}, \frac{\partial}{\partial z_{j}}\right)=\delta_{i j}$ partout. Avec le même argument que ci-dessus, on montre facilement que les $g\left(\frac{\partial}{\partial y_{i}}, \frac{\partial}{\partial y_{j}}\right)$ et les $g\left(\frac{\partial}{\partial y_{i}}, \frac{\partial}{\partial z_{k}}\right)$ ne dépendent pas des coordonnées $x_{i}$. D'où le

Théorème 5.27. Soit $(M, g)$ une variété pseudo-riemannienne de signature $(r, r+n)$ et de dimension $m=n+2 r$ dont le groupe d'holonomie restreint laisse invariant un sous-espace isotrope maximal. Alors il existe des coordonnées $\left(x_{1}, \ldots, x_{r}, y_{1}, \ldots, y_{n}, z_{1}, \ldots, z_{r}\right)$ dans lequel la matrice $S$ de $g$ a la forme réduite suivante:

$$
S=\left(\begin{array}{ccc}
0 & 0 & I_{r} \\
0 & B & C \\
I_{r} & { }^{t} C & D
\end{array}\right)
$$

où $B, C$ et $D$ sont des matrices d'ordre respectivement $(n, n),(n, r)$ et $(r, r)$ telles que les matrices $B$ et $C$ ne dépendent pas des coordonnées $x_{i}$. 


\section{Autres exemples}

Contrairement au cas lorentzien $(r=1)$ (voir $[\mathbf{3}]$ ), il n'y a jusqu'à présent aucune obstruction pour qu'un groupe vérifiant les conclusions du Théorème 3.19 soit groupe d'holonomie restreint d'une variété indécomposable non-irréductible. Nous donnerons dans la suite des exemples de telles variétés.

Soit $\mathcal{H}$ une sous-algèbre de $\mathcal{G}$ qui satisfait les conclusions du Théorème 3.19. Nous distinguerons quatre cas:

Type 1: $\mathcal{H}$ contient $\mathcal{U}^{\prime}$ et $\mathcal{P} \oplus \mathcal{B}$. Donc $\mathcal{H}=\mathcal{U}^{\prime} \oplus \mathcal{K}^{\prime} \oplus \mathcal{P} \oplus \mathcal{B}$. Cet exemple apparaît toujours en dimension $4\left(\mathcal{K}^{\prime}=\mathcal{P}=0\right)$ et en dimension $5\left(\mathcal{K}^{\prime}=0\right)$. Le sous-groupe connexe engendré par $\mathcal{P} \oplus \mathcal{B}$ est

$$
P B=\left\{\left(\begin{array}{ccc}
I_{2} & { }^{t} Y & \frac{1}{2}^{t} Y \cdot Y+\delta J \\
0 & I_{n} & Y \\
0 & 0 & I_{2}
\end{array}\right), Y \in \mathcal{P}, \delta \in \mathbb{R}\right\} \simeq \mathbb{R}^{d+1}
$$

avec $d$ la dimension de $\mathcal{P}$. $P B$ est un sous-groupe nilpotent fermé de $G$. Le sous-groupe connexe $U^{\prime}$ de $G L(2, \mathbb{R})$ engendré par $\mathcal{U}^{\prime}$ est fermé, si et seulement si son radical $R$ l'est. Or $R$ ne peut être à une conjuguaison près dans $G L(2, \mathbb{R})$ que contenu dans $S O(2, \mathbb{R})+\mathbb{R}$, ou contenu dans le groupe

$$
\left\{\left(\begin{array}{ll}
\alpha & \gamma \\
0 & \beta
\end{array}\right), \alpha, \beta>0 \text { et } \gamma \in \mathbb{R}\right\}
$$

d'où $U^{\prime}$ est fermé. Or le sous-groupe connexe $K^{\prime}$ de $S O(n, \mathbb{R})$ engendré par $\mathcal{K}^{\prime}$ n'est pas nécessairement fermé si $n \geq 4$. Donc $H=U^{\prime} K^{\prime} P B$ aussi. Mais si $K^{\prime}$ est le groupe d'holonomie (restreint) d'une métrique riemannienne $g$ définit sur $\mathbb{R}^{m}=\left\{x_{3}, \ldots, x_{n+2}\right\}, K^{\prime}$ est nécessairement complètement réductible. On considère la métrique de signature $(2,2+n)$ définit sur $\mathbb{R}^{m}(m=n+4)$ par

$$
h=2 d x_{1} d x_{n+3}+2 d x_{2} d x_{n+4}-g+f_{1} d x_{n+3}^{2}+f_{2} d x_{n+4}^{2}
$$

avec $f_{1}$ et $f_{2}$ deux fonctions suffisament génériques indépendantes des variables $x_{1}$ et $x_{2}$. En utilisant le Théorème d'Ambrose-Singer [2] et le Théorème 5.27, on montre avec un calcul simple que $K^{\prime} \cdot N B$ est le groupe d'holonomie (restreint) de la métrique $h$ ( $N B$ est le sous-groupe connexe engendré par $\mathcal{N} \oplus \mathcal{B})$. 
On considère maintenant la métrique de signature $(2,6)$ définit sur $\mathbb{R}^{8}$ par

$$
\begin{aligned}
h=2 d x_{1} d x_{7}+ & 2 d x_{2} d x_{8}-\sum_{i=3}^{6} d x_{i}^{2}+2 x_{4} x_{8} d x_{3} d x_{7}+2 r x_{6} x_{8} d x_{5} d x_{7} \\
& +2 x_{3} x_{7} d x_{4} d x_{8}+2 r x_{5} x_{7} d x_{6} d x_{8}+\sum_{j=3}^{8} x_{j}^{2} d x_{7} d x_{8} .
\end{aligned}
$$

Avec un calcul simple et avec le même argument que ci-dessus, on peut montrer que son groupe d'holonomie (restreint) est de type 1 (avec $\mathcal{K}^{\prime}=$ $\mathcal{U}^{\prime}=0$ ) et il est fermé si et seulement si, $r$ est rationnel.

Type 2: $\mathcal{H}$ contient $\mathcal{P} \oplus \mathcal{B}$ mais ne contient pas $\mathcal{U}^{\prime}$. Donc $\mathcal{H}=$ $\mathcal{L} \oplus \mathcal{P} \oplus \mathcal{B}$, avec $\mathcal{L}=\left\{(U, A, 0,0), U \in \mathcal{U}^{\prime}, A \in \mathcal{K}^{\prime}\right\}$ une sous-algèbre de $\mathcal{U}^{\prime} \oplus \mathcal{K}^{\prime}$. Cet exemple apparaît en dimension $6\left(\mathcal{K}^{\prime}=S O(2)\right)$ ou plus. Si on note $L$ le sous-groupe connexe de $G$ engendré par $\mathcal{L}$, il n'est pas nécessairement fermé. Il suffit de choisir $\mathcal{L}$ engendrée par $(r J, J)$ avec $r \neq 0$ une constante rationnelle. Donc $H=L P B$ n'est pas nécessairement fermé aussi. On considère la métrique de signature $(2,4)$ définit sur $\mathbb{R}^{6}$ par

$$
\begin{aligned}
h=2 d x_{1} d x_{5}+2 d x_{2} d x_{6}- & d x_{3}^{2}-d x_{4}^{2}+2 x_{4} x_{6} d x_{3} d x_{5} \\
& +\left(-2 x_{1} x_{6}+2 r x_{2} x_{5}+x_{3}^{2}+x_{4}^{2}\right) d x_{5} d x_{6} .
\end{aligned}
$$

Avec un calcul simple, on peut montrer que son groupe d'holonomie (restreint) est de type 2. Et il est fermé.

Type 3: $\mathcal{H}$ vérifie 2.1) du Théorème 3.19. Donc $\mathcal{H}=\mathcal{L}_{U} \oplus \mathcal{L}_{D} \oplus \mathcal{L}_{C} \oplus$ $\mathcal{P} \oplus \mathcal{B}$, avec $\mathcal{L}_{U}=\left\{\left(U, \varphi(U), \psi\left((U, \varphi(U))+\phi((U, \varphi(U)), 0), U \in \mathcal{U}^{\prime}\right\}\right.\right.$, $\mathcal{L}_{D}=\{(0, B, \psi(B), 0), B \in \mathcal{D}\}, \mathcal{L}_{C}=\{(0, A, \psi(A)+\phi(A), 0), A \in \mathcal{C}\}$ (pour les notations voir le théorème). Cet exemple appara^ $\{$ it en dimensions $\geq 7$. Si par exemple $\mathcal{U}^{\prime}=\mathcal{D}=0, \psi=0, \mathcal{C}$ engendrée par $A=\left(\begin{array}{cc}J & 0 \\ 0 & r J\end{array}\right), r \in \mathbb{R}^{*}$ et $\phi(A)=(1,0)$, alors $H$ est fermé si et seulement si, $r$ est rationnel. En plus, on peut montrer que c'est le groupe d'holonomie (restreint) de la métrique de signature $(2,7)$ définit sur $\mathbb{R}^{9}$ par

$$
\begin{array}{r}
h=2 d x_{1} d x_{8}+2 d x_{2} d x_{9}-\sum_{i=3}^{7} d x_{3}^{2}+2 x_{4} x_{9} d x_{3} d x_{8}+\frac{2}{r} x_{6} x_{9} d x_{5} d x_{8} \\
-2 x_{8}^{2} d x_{4} d x_{9}+\sum_{i=3, i \neq 7}^{7} x_{i}^{2} d x_{8} d x_{9} .
\end{array}
$$


Type 4: $\mathcal{H}$ vérifie 2.2) ou 2.3) ou 2.4) du Théorème 3.19. Ces exemples apparaîssent seulement en dimensions $\geq 6$. Nous donnerons des exemples dans un futur article.

De ce qui précède, on obtient

Théorème 6.28. En dimensions $\leq 5$ le groupe d'holonomie restreint d'une métrique de signature $(2,2+n)$ qui laisse invariant au moins un plan totalement isotrope et qui contient $B$ est fermé. Il existe des exemples de variétés pseudo-riemanniennes de signature $(2,2+n)$ pour $n \geq 2$ dont les groupes d'holonomie restreints ne sont pas fermés.

\section{Références}

1. D. V. AlekseevskiI, Riemannian manifolds with exceptional holonomy groups, Funktsional. Anal. i Prilozhen. 2(2) (1968), 1-10; English translation: Functional Anal. Appl. 2 (1968), 97-105.

2. W. Ambrose et I. M. Singer, A theorem on holonomy, Trans. Amer. Math. Soc. 79 (1953), 428-443.

3. L. Berard Bergery et A. IKemakhen, On the holonomy of Lorentzian Manifolds, Proceedings of Symposia in Pure Mathematics 54, Part 2 (1993), 27-40.

4. L. Berard Bergery et A. Ikemakhen, Sur l'holonomie des variétés pseudo-riemanniennes de signature $(n, n)$, Bull. Soc. Math. France 125 (1997), 93-114.

5. M. Berger, Sur les groupes d'holonomie des variétés à connexion affine et des variétés riemanniennes, Bull. Soc. Math. France $\mathbf{8 3}$ (1955), 279-330.

6. M. Berger, Les espaces symétriques non compacts, Ann. Sci. École Norm. Sup. 74 (1957), 85-177.

7. A. L. Besse, "Einstein manifolds," Springer-Verlag, Berlin-Heidelberg-New York, 1987.

8. R. Brown et A. Gray, Riemannian manifolds with holonomy group Spin(9), in Diff. Geometry in honor of K. Yano, Kinokuniya, Tokyo (1972), 41-59.

9. R. L. Bryant, Metrics with exceptional holonomy, Ann. of Math. 126(2) (1987), 525-576.

10. R. L. Bryant, Classical, Exceptional and exotic holonomies: a statuts report, Actes de la table Ronde Table de la Géometrie Différentielle en l'honneur de M. Berger, Collection Séminaire et Congrès de la Société Mathématique de France 1 (1996), 93-165. 
11. R. L. Bryant et S. M. Salamon, On the construction of some complete metrics with exceptional holonomy, Duke Math. J. 58(3) (1989), 829-850.

12. M. Cahen et M. Parker, Sur des classes d'espaces pseudoriemanniens symétriques, Bull. Soc. Math. Belg. 22 (1970), 339-354.

13. M. CAhen ET M. PARKER, Pseudo-Riemannian symmetric spaces, Mem. Amer. Math. Soc. 229 (1980).

14. M. Cahen et N. Wallach, Lorentzian symmetric spaces, Bull. Amer. Math. Soc. 79 (1970), 585-591.

15. E. Calabi, Métriques kähleriennes et fibrés holomorphes, Ann. Sci. École Norm. Sup. 12 (1979), 269-294.

16. Q. S. Chi, S. Merkulov et L. Schwachhöfer, On the existence of infinite series of exotic holonomies, Invent. Math. 126(2) (1996), 391-411.

17. A. IKEMAKHEN, Examples of indecomposable non-irreducible Lorentzian manifolds, Ann. Sci. Math. Québec 20(1) (1996), 53-66.

18. S. Merkulov, Affine connections on involutive $G$-structures, in "Geometry and Physics," Lecture notes in Pure and Applied Mathematics 184, 1995, pp. 261-274.

19. D. Montgomery et H. SAmelson, Transformation groups of spheres, Ann. of Math. 44 (1943), 454-470.

20. M. PARKER, Classes d'espaces pseudo-riemanniennes symétriques de signature (2,n), C. R. Acad. Sci. Paris 272 (1971), 882-884.

21. S. Salamon, "Riemannian geometry and Holonomy groups," Pitman Research Notes in Maths 201, Longman Scientific \& Technical, London, 1989.

22. L. J. SchwachHöFER, Connections with exotic holonomy, Trans. Amer. Math. Soc. 345(1) (1994), 293-321.

23. L. SCHWACHHÖFER, On affine connections whose holonomy is a tensor representation, Z. Anal. Anwendungen 16(1) (1997), 119-130.

24. J. Simons, On the transitivity of holonomy systems, Ann. of Math. 76 (1962), 213-234.

25. H. Wu, On the de Rham decomposition theorem, Illinois J. Math. 8 (1964), 291-311. 
26. H. Wu, Holonomy groups of indefinite metrics, Pacific J. Math. 20 (1967), 351-392.

\author{
Département de Mathématiques \\ Faculté des sciences et techniques$$
\text { Université Cadi-Ayyad }
$$$$
\text { B. P. } 618 \text { Guéliz-Marrakech }
$$ \\ MAROC \\ e-mail: fstg@cybernet.net.ma
}

Primera versió rebuda el 8 de juliol de 1997, darrera versió rebuda el 21 de juliol de 1998 